\title{
Wireless Mesh Network Capacity Achievable Over the CSMA/CA MAC
}

\author{
Yu Cheng, Senior Member, IEEE, Hongkun Li, Student Member, IEEE, Peng-Jun Wan, Member, IEEE, and \\ Xinbing Wang, Senior Member, IEEE
}

\begin{abstract}
This paper presents a theoretical analysis of the maximum throughput of a wireless mesh backhaul network that is achievable over a practical carrier sense multiple access with collision avoidance (CSMA/CA) medium access control (MAC) protocol. We resort to the multicommodity flow (MCF) formulation augmented with the conflict-graph constraints, whereas we use a novel approach to take into account the collision overhead in the distributed CSMA/CA MAC. Such overhead due to random access has been ignored by existing MCF-based capacity studies, which assume impractical centralized scheduling and result in aggressive capacity planning, which is unachievable over the CSMA/CA MAC. This paper makes the following three main contributions: 1) we develop a generic method of integrating the CSMA/CA MAC analysis with the MCF formulation for optimal network capacity analysis, which readily generates an upper bound of the network throughput; 2) we define a new concept of CSMA/CA clique and theoretically study its relationship to a CSMA/CA area in terms of throughput; and 3) using the CSMA/CA clique as a tool, we derive a lower bound of the network throughput achievable over the CSMA/CA MAC by clique-based MCF formulation. NS-2 simulation results are presented to demonstrate the tightness of the upper and lower bounds that are newly developed, compared to those based on the MCF formulation assuming a slotted system and centralized scheduling.
\end{abstract}

Index Terms-Carrier sense multiple access with collision avoidance (CSMA/CA), conflict graph, multicommodity flow (MCF), network capacity, wireless mesh network.

\section{INTRODUCTION}

A WIRELESS mesh backhaul network connects access networks through multihop wireless links to the Internet backbone. The network capacity of importance to a backhaul network is the total throughput that traverses the given set of ingress/egress edge nodes. The maximum network capacity

Manuscript received November 23, 2011; revised May 1, 2012; accepted June 3, 2012. Date of publication June 12, 2012; date of current version September 11, 2012. The work of Y. Cheng and H. Li was supported in part by the National Science Foundation (NSF) under Grant CNS-0832093 and Grant CNS-1053777. The work of P. Wan was supported in part by NSF under Grant CNS-0916666. The work of X. Wang was supported in part by NSF China under Grant 60702046 and Grant 60832005. This paper was presented in part at the 29th IEEE International Conference on Computer Communications, 2010. The review of this paper was coordinated by Dr. T. Taleb.

Y. Cheng and H. Li are with the Department of Electrical and Computer Engineering, Illinois Institute of Technology, Chicago, IL 60616-3793 USA (e-mail: cheng@iit.edu; hli55@iit.edu).

P.-J. Wan is with the Department of Computer Science, Illinois Institute of Technology, Chicago, IL 60616 USA (e-mail: wan@cs.iit.edu).

$\mathrm{X}$. Wang is with the Department of Electronic Engineering, Shanghai Jiaotong University, Shanghai 200240, China (e-mail: xwang8@ @stu.edu.cn).

Color versions of one or more of the figures in this paper are available online at http://ieeexplore.ieee.org.

Digital Object Identifier 10.1109/TVT.2012.2204411 is normally coupled with optimal routing and scheduling to form a network dimensioning issue [1], [2]. One fundamental issue in the multihop wireless networks, which is different from the wireline case, is that the neighboring hops along a path, when transmitting over the same spectrum, have to contend for channel access and cannot transmit at the same time. The conflict graph or contention graph is a popular tool for modeling the interference among different wireless links [3]-[5]. The main-thread approach for wireless network dimensioning is to apply a multicommodity flow (MCF) formulation, augmented with constraints derived from the conflict graph [1]-[3], [6], [7].

The conflict-graph constraints can be defined based on either the independent set or the clique [3]. The existing MCFbased dimensioning studies mainly focus on the independentset-based formulation, where the MCF solution can generate an optimal centralized scheduling (under the assumption of a synchronized slotted system): the maximal independent sets (MISs) take turns in grabbing the channel for data transmission, with the proportion of transmission time for each set determined by the MCF solution. However, the maximum throughput based on the optimal centralized scheduling is not achievable for a network that applies a distributed carrier sense multiple access with collision avoidance (CSMA/CA) protocol for medium access control (MAC), e.g., the IEEE 802.11 distributed coordination function (DCF). The main reason for this is that collisions are inevitable for the CSMA/CA MAC due to its nature of random access, which leads to reduced channel utilization. Many MCF capacity studies [3], [8], [9] claim that the impact of 802.11 is considered, but it is, in fact, treated as a specific protocol interference model to define the conflict graph for centralized scheduling. Therefore, it is of critical importance to develop analytical tools for determining the maximum network throughput that is achievable over a practical CSMA/CA MAC. This paper has three main contributions along such a direction.

First, we develop a generic method of integrating CSMA/CA MAC analysis with the MCF formulation, and therefore, the random access collision overhead could be incorporated into the network capacity analysis. Note that it is hard to continue with the independent-set model to study the impact of channel collisions, because the purpose of an independent set is to identify links that are located far away from each other and, thus, can simultaneously transmit without interference. Therefore, in this paper, we resort to the clique-based method. A clique contains links that mutually conflict with each other. By considering each clique as one single-hop area, we reveal 
that applying the channel utilization analysis for a single-hop CSMA/CA area to each clique gives a generic method of correlating the MCF formulation with the CSMA/CA MAC modeling. To the best of our knowledge, this is the first effort in this direction. In [10], we theoretically demonstrate that the maximum normalized throughput within a single-hop area, denoted as $R_{g}^{*}$ and called the optimal operation point, is determined by the CSMA/CA nature and is independent of the MAC implementation details. In the multihop context, the protocol-independent throughput analysis implies that the total channel utilization within a maximal clique is at most $R_{g}^{*}<1$, whereas the existing MCF formulations consider that the channel utilization within a maximal clique can be up to 1 for computing a capacity upper bound [3], [11]. With the modified clique utilization of $R_{q}^{*}$, a tighter upper bound of the network throughput over the CSMA/CA MAC can be obtained.

The second main contribution of the paper is a theoretical study of the relationship between a CSMA/CA area and its associated CSMA/CA clique. A circle with a diameter of carrier-sensing range is defined as a CSMA/CA area. A CSMA/CA clique consists of the links incident to the nodes in the CSMA/CA area. The CSMA/CA clique is a newly proposed concept, different from the maximal clique. Note that integrating the CSMA/CA optimal operation point analysis with the clique-based MCF formulation to compute achievable throughput is not only a combination issue. For convenience, we define two nodes within the carrier sensing range of each other as interfering nodes, which could also be stated as there exists interference between the two nodes. The clique focuses on the interference among links, whereas a CSMA/CA area focuses on the interference among sending nodes in spite of the receiver nodes. We theoretically prove that the total normalized throughput over a CSMA/CA clique can be transformed to that over a CSMA/CA area and can thus be also constrained by the maximum throughput $R_{g}^{*}$. We further prove that the total throughput constraint of $R_{g}^{*}$ can ensure the achievability of any heterogeneous rate allocation within a CSMA/CA area, with the transmission opportunity (TXOP)-based [12] differentiation scheme applied. Such theoretical results provide a foundation for deriving the lower bound of the network throughput.

The third main contribution of this paper is the theoretical proof of a sufficient condition that guarantees the achievability of a network flow allocation over the CSMA/CA MAC. The essence of enhancing the clique-based MCF formulation to generate feasible network throughput is to take into account the interference among different cliques, whereas the basic clique constraints themselves (well known as necessary conditions) simply describe the interference within each clique. By leveraging our new tools of CSMA/CA area and CSMA/CA clique, we prove, for any given mesh network, that one sufficient condition that ensures the MCF capacity solution achievable over the CSMA/CA MAC (i.e., generating a lower bound) is the normalized throughput over any maximal CSMA/CA clique $\leq \gamma R_{g}^{*}$, with the TXOP differentiation scheme applied for heterogeneous rate allocation. The scaling factor $\gamma(\leq 1)$ is determined by the maximum number of network nodes that may interfere with a tagged sending node and the minimum number of network nodes covered by a maximal CSMA/CA area. We further prove that such a sufficient condition $\left(\leq \gamma R_{g}^{*}\right)$ also applies to maximal cliques. In particular, it is proved that $\gamma=1 / 4$ for a uniform network in which all the nodes are uniformly or regularly distributed. Compared to the upper bound, the lower bound indicates that the sufficient clique condition developed for the CSMA/CA MAC can ensure a feasible capacity, which is at least $\gamma$ (e.g., 1/4 for a uniform network) of the maximum capacity achievable over the CSMA/CA or $\gamma R_{g}^{*}$ of the maximum capacity under the optimal centralized scheduling. The factor $\gamma$ is often called the capacity efficiency ratio of the algorithm.

The MCF problem over wireless mesh networks is NP-hard in general [3], [8]. Capacity bound analysis is a typical approach for dealing with such hard problems [8], [11]. We notice that the lower bound indicated by a capacity efficiency ratio of $\gamma=1 / 4$ is conservative. This is because the clique constraints, by nature, only model the interference in local areas; for the local analysis to be applicable over the whole network, the worst case modeling of the interference has to be applied. Note that the efficiency ratio of $1 / 4$ that was obtained in this paper is an improved lower bound compared to the existing studies. In a slotted system, both capacity-planning algorithms [13] and distributed-scheduling algorithms [14] have been studied, which can guarantee a capacity efficiency ratio of $1 / \mathcal{K}$; the variable $\mathcal{K}$ denotes the network interference degree, and it has been shown that $(1 / \mathcal{K})=(1 / 8)$ under the 802.11-like interference model [15]. In [16], a capacity efficiency ratio of $1 / 6$ is derived for a centralized greedy maximal scheduling algorithm under the 802.11-like interference model. We would like to emphasize that almost all of the existing capacity efficiency ratio studies assume a slotted system.

To the best of our knowledge, this paper is the first theoretical capacity analysis over the CSMA/CA MAC in the network-dimensioning context based on the MCF formulation. The significant advantage of our capacity analysis is that the MCF capacity planning results can directly be deployed over the distributed CSMA/CA MAC and the complex scheduling issue is totally avoided. The work in [11] studied sufficient clique constraints for computing achievable MCF capacity, but assuming centralized scheduling. Moreover, the analysis in [11] could only prove the existence of a scheduling mechanism, but how to find a distributed scheduling mechanism was indicated as a well-known open issue. In this paper, we will present simulation results to explicitly demonstrate that the MCF capacity planning results under a centralized scheduling (e.g., MIS-based scheduling) is not feasible over a distributed CSMA/CA MAC. Thus, arbitrarily applying MCF capacity planing over CSMA/CA MAC without thorough consideration is unsatisfying.

The remainder of this paper is organized as follows. Section II reviews more related work. Section III summarizes the CSMA/CA optimal operation point analysis. Section IV describes the MCF formulation for a wireless mesh network. Section V investigates the upper and lower bounds of the network capacity over the CSMA/CA MAC. Section VI presents some ns-2 simulation results to demonstrate the tightness of the upper and lower bound that are newly developed. Section VII gives the concluding remarks. 


\section{RELATED WORK}

Note that there is a thread of capacity analysis to study the asymptotic scaling laws of the network capacity versus the network size; refer to [17]-[20] and the references therein. In this paper, however, we focus on computing the achievable capacity of a given wireless mesh network.

There are two models - the protocol interference model and the physical interference model [3], [17]—for defining the conditions for a successful transmission. Under the physical interference model, also known as the signal-to-interferenceplus-noise-ratio (SINR) model, a transmission is successful if and only if the SINR at the intended receiver exceeds a specified threshold. Although the physical model is an accurate description of the real system, the SINR calculation is a nonconvex function with respect to the transmission power, which incurs high computational complexity in solving a capacity optimization problem under the physical model. To circumvent the complexity issue, the protocol interference model has widely been used in the wireless networking research community. Under the protocol interference model, a successful transmission occurs when a node falls inside the communication range of its intended transmitter and out of the interference range of other nonintended transmitters. A recent paper [21] discusses how the interference range is properly set so that a physical interference model can accurately be transformed to a protocol interference model. We adopt the protocol interference model in this paper.

The MCF problem under the protocol interference model is, however, NP-hard in general [3]. A rigorous NP-hardness proof is given in [8]. The lower and upper bounds of such an NP-hard problem can be computed by solving a linearprogramming (LP) problem under constraints that are based on the MISs and the maximal cliques [3], respectively. Searching for all the MISs or maximal cliques is still NP-hard. Even given all the MISs or maximal cliques, the LP MCF problem has to be solved with exponentially many constraints. Considering the high computational complexity, there are many efforts in developing polynomial approximation algorithms [6], [8], [9] for the MCF problem over wireless networks.

In this paper, our focus is not on approximation algorithms. Instead, we study how we can enhance the MCF formulation and extend the conflict-graph-based constraints to generate the capacity region, particularly an achievable lower bound, over the CSMA/CA MAC. Many MCF capacity studies [3], [8], [9] claim that the impact of 802.11 is considered, but it is, in fact, treated as a specific protocol interference model to define the conflict graph for centralized scheduling. The fundamental contribution of this paper is to derive necessary and sufficient conditions for MCF flow allocation, which take the CSMA/CA randomness nature into account. Some of our preliminary results have been published in [22], where the sufficient condition that ensures an achievable capacity over the CSMA/CA MAC was given only in a specific case, i.e., in the uniform networks. In this paper, we develop the general sufficient condition for an arbitrarily given wireless mesh network. Moreover, all the theoretical proofs (some of which were omitted in [22]) are presented in detail.
Some recent works have paid attention to the wireless network performance over distributed MAC protocols. Analytical models [23], [24] have been developed to compute per-flow throughput for a multihop 802.11 network, given the input traffic load and routing paths. However, such a model could not be applied to analyze the maximum capacity region. The recent work on throughput-optimal algorithms, i.e., [25]-[27], and the references therein focus on developing distributed implementations by applying decomposition techniques to the MCF optimization formulation, but the derived distributed algorithms again assume a slotted system and cannot directly be applied to the CSMA/CA MAC with random access. The interesting theoretical studies in [28] and [29] indicate that CSMA-based MAC with an ideal adaptive backoff mechanism can achieve the optimal utility by sacrificing the short-term fairness, but the results do not provide insights into how to compute the achievable capacity region over a given practical CSMA/CA MAC protocol.

The capacity bound analysis that is presented in this paper is essentially related to $R_{q}^{*}$, the optimal operation point (i.e., the maximum normalized throughput) of a single-hop CSMA/CA area. The analysis can readily be extended to incorporate the capture effect [30], [31]. According to the standard IEEE 802.11 DCF protocol, simultaneous transmissions result in a collision where no transmission is considered successful. However, in practice, a certain transmission involved in a collision situation could be successful if its power at the receiver is stronger than other interfering transmissions by a reasonable threshold. Such a phenomenon is called capture effect, which could considerably improve the throughput over the IEEE 802.11 DCF. The existing studies have shown that the capture effect can mathematically be modeled with a conditional probability of successful transmission $p_{\text {capt }}$, given that a collision happens [30], [31]. In Section III, we will show that the capture probability $p_{\text {capt }}$ leads to a higher optimal operation point $R_{g}^{*}$. All other analysis in this paper still applies to such improved $R_{g}^{*}$ due to the capture effect.

Multi-radio multi-channel (MR-MC) wireless networks have attracted much attention in recent few years [13], [32]. With MR-MC communications, the network capacity can significantly be improved by simultaneously exploiting multiple nonoverlapping channels through different radio interfaces and mitigating interferences through proper network configuration. In [33], we develop a novel tool of a multidimensional conflict graph (MDCG). Resorting to the MDCG, we show that MR-MC wireless networks and single-radio-single-channel (SR-SC) wireless networks can be studied with a uniformed MCF framework, which paves the way for extending the CSMA/CA capacity analysis developed in this paper to the MR-MC context. Aiming at further capacity increase, there are studies [34], [35] (including our work [36]) that exploit the partially overlapping channels in addition to the nonoverlapping channels. However, the physical interference model (or the SINR model) has to be involved to properly describe the interference between two simultaneous transmissions over partially overlapping channels. Developing efficient algorithms to exploit the partially overlapping channels is still an open challenge, even in the context of centralized scheduling. Dealing with multiple channels, either nonoverlapping or partially 
overlapping, is not a trivial issue. As a future topic, we will study how CSMA/CA capacity analysis can be extended to multichannel scenarios.

\section{Optimal Operation Point}

We developed a protocol-independent analysis in [10], which reveals that the family of CSMA/CA protocols share the same optimal operation point where the maximum protocol capacity is achieved. The protocol-independent analysis is inspired by the concept of virtual slot [37]-[39]. Observed at the timescale of a virtual slot, all the CSMA/CA protocols show the same behavior that the channel alternates among the statuses of idling, successful transmission, and collision. Moreover, it is proved in [10] that the traffic arrival process at each virtual slot can be accurately described or well approximated by a Poisson random variable, when channel accesses from different users are independent (e.g., in 802.11 DCF) or weakly correlated (e.g., in an 802.15.4 contention access period), respectively. If the size variation of different types of virtual slots were ignored, the virtual-slot system would stochastically behave, similar to a slotted ALOHA protocol [40]. Therefore, the well-known S-G analysis [40] for slotted ALOHA can be extended into a virtualslot-based $S-G$ analysis (VS S-G).

Let $G$ denote the Poisson traffic load in a slot. The probability of seeing an idle (a successful transmission) slot $p_{d}\left(p_{s}\right)$ equals the probability that zero (a single) transmission trail happens at a slot. That is, $p_{d}=e^{-G}, p_{s}=G e^{-G}$, and the probability of seeing a collision slot $p_{c}=1-G e^{-G}-e^{-G}$. Let $\sigma, T_{s}$, and $T_{c}$ denote the lengths of virtual slots associated with channel idling, successful transmission, and collisions, respectively. The steady-state channel utilization $R_{s}$ can be computed as

$$
R_{s}=\frac{p_{s} T_{s}}{p_{d} \sigma+p_{s} T_{s}+p_{c} T_{c}} .
$$

To obtain the maximum channel utilization (equivalently, the maximum MAC protocol capacity), we can determine the optimal workload $G^{*}$ according to

$$
\left.\frac{d}{d G} R_{s}\right|_{G=G^{*}}=0
$$

which then gives

$$
\begin{aligned}
e^{G^{*}} & \left(1-G^{*}\right) \\
& =\frac{G^{*}\left[T_{s}\left(1-G^{*}\right)+G^{*} T_{c}-\sigma\right]}{e^{-G^{*}} \sigma+G^{*} e^{-G^{*}} T_{s}+\left(1-G^{*} e^{-G^{*}}-e^{-G^{*}}\right) T_{c}}
\end{aligned}
$$

after some manipulation. The value of $G^{*}$ can numerically be solved based on (3).

Let $p_{d}^{*}, p_{s}^{*}$, and $p_{c}^{*}$ denote the values of the probabilities under the optimal workload $G^{*}$. The maximum channel utilization can be obtained as

$$
R_{s}^{*}=\frac{p_{s}^{*} T_{s}}{p_{d}^{*} \sigma+p_{s}^{*} T_{s}+p_{c}^{*} T_{c}} .
$$

Let $L_{p}$ denote the payload size of a packet and $C$ denote the spectrum bandwidth. The maximum normalized throughput $R_{g}^{*}$ can then be computed as

$$
\begin{aligned}
R_{g}^{*} & =\frac{p_{s}^{*} L_{p}}{C\left(p_{d}^{*} \sigma+p_{s}^{*} T_{s}+p_{c}^{*} T_{c}\right)} \\
& =\frac{R_{s}^{*} t_{p}}{T_{s}}
\end{aligned}
$$

where $t_{p}=\left(L_{p} / C\right)$ represents the effective transmission time within each successful period. The maximum normalized throughput indicates the optimal operation point.

The throughput $R_{g}^{*}$ at the optimal operation point is achievable. Assume that $N$ nodes contend for the channel according to 802.11 DCF and all nodes are homogeneously configured. The optimal work load $G^{*}$ at the optimal operation point can then be mapped to the channel access probability of each node in an idle slot as $\tau=G^{*} / N .{ }^{1}$ The channel access probability $\tau$ can further be mapped to an initial contention window value $W_{c}$ for the exponential backoff operation through detailed MAC analysis [39, eqs. (2)-(5)].

Remark 1: We can easily extend the optimal operation point analysis to incorporate the capture effect. With $p_{\text {capt }}$ denoting the capture probability, given a collision, the probability of having a successful transmission in a slot is then increased to $p_{s}+p_{\text {capt }} p_{c}$. Correspondingly, the steady-state channel utilization under the capture effect is then increased to $R_{s, c a p t}$ as

$$
R_{s, \text { capt }}=\frac{\left(p_{s}+p_{\text {capt }} p_{c}\right) T_{s}}{p_{d} \sigma+\left(p_{s}+p_{\text {capt }} p_{c}\right) T_{s}+\left(1-p_{\text {capt }}\right) p_{c} T_{c}} .
$$

The optimal workload $G^{*}$ can then be determined according to $\left.\frac{d}{d G} R_{s, \text { capt }}\right|_{G=G^{*}}=0$. With $G^{*}$, we can then follow the aforementioned procedure to obtain the optimal operation point under the capture effect.

\section{Multicommodity Flow Formulation}

We consider a single-channel wireless backhaul network, represented as a directed graph $\mathcal{G}(\mathcal{N}, \mathcal{L})$ with node set $\mathcal{N}$ and link set $\mathcal{L}$. Each node $n_{i}$ has a communication range $\chi_{i}$ and a potentially larger interference range $\chi_{i}^{\prime}$. There is a directed link $l_{i j}$ from node $n_{i}$ to node $n_{j}$ if $d_{i j} \leq \chi_{i}$. The capacity of link $l_{i j}$ is denoted as $C_{i j}$ and assumed to be time invariant. Bidirectional transmissions between two nodes are described by two directed links, one for each direction. We adopt the protocol interference model.

\section{A. Basic MCF Formulation}

Let $(\nu, \eta)$ denote an ingress/egress pair of the backhaul network and $\Lambda$ denote the set of ingress/egress pairs. We further use $x_{\nu \eta}(i, j)$ to denote the portion of $n_{\nu}$-to- $n_{\eta}$ traffic flow, which traverses the link $l_{i j}$. The classic MCF formulation, without considering the wireless interference, is expressed as

$$
\max \sum_{(\nu, \eta)} \sum_{l_{\nu i} \in \mathcal{L}} x_{\nu \eta}(\nu, i)
$$

\footnotetext{
${ }^{1}$ For 802.11 , the access process in each idle slot is binomially distributed with parameters $N$ and $\tau$. When $N$ is reasonably large, the binomial distribution converges to a Poisson random variable with rate $G=N \tau$ [10].
} 
subject to

$$
\begin{gathered}
\sum_{l_{j i} \in \mathcal{L}} x_{\nu \eta}(j, i)=\sum_{l_{i j} \in \mathcal{L}} x_{\nu \eta}(i, j) \\
\forall(\nu, \eta) \in \Lambda \text { and } n_{i} \in \mathcal{N} /\left\{n_{\nu}, n_{\eta}\right\} \\
\sum_{l_{i \nu} \in \mathcal{L}} x_{\nu \eta}(i, \nu)=0 \quad \forall(\nu, \eta) \in \Lambda \\
\sum_{l_{\eta i} \in \mathcal{L}} x_{\nu \eta}(\eta, i)=0 \quad \forall(\nu, \eta) \in \Lambda \\
x_{\nu \eta}(i, j) \geq 0 \quad \forall l_{i j} \in \mathcal{L} \text { and }(\nu, \eta) \in \Lambda \\
\sum_{(\nu, \eta)} x_{\nu \eta}(i, j) \leq C_{i j} \quad \forall l_{i j} \in \mathcal{L} .
\end{gathered}
$$

The formulation states that we aim at maximizing the aggregate throughput that traverses all the ingress/egress pairs, with the following five constraints.

1) Flow conservation constraint (7). At every node, except for the source and the destination, the amount of incoming flow associated with a certain ingress/egress pair equals the amount of outgoing flow for that ingress/egress pair.

2) Source constraint (8). For each ingress/egress pair, the incoming flow to the source node is 0 .

3) Destination constraint (9). The outgoing flow from the destination node is 0 .

4) Nonnegative constraint (10). The amount of flow allocation should be nonnegative.

5) Link constraint (11). The total amount of flow on a link cannot exceed the link capacity.

Note that the MCF problem has different flavors. The optimization problem described in (6) is specifically called the maximum multiflow (MMF) problem. The MMF problem targets at maximizing the total throughput over the whole network but does not provide any guarantee on the minimum throughput between a certain pair of source and destination nodes or the fairness among different commodity flows. Another example of the MCF problem with the fairness property is the maximum concurrent multiflow (MCMF) problem, which targets at maximizing the minimal fraction of the flow of each commodity to its demand. With regard to the achievable capacity analysis over CSMA/CA, the theoretical treatments to MMF and MCMF are slightly different. In the following section, we mainly develop the theoretical analysis based on MMF, and then, we discuss the MCMF problem in Section V-D.

\section{B. Conflict Graph Constraints}

To incorporate wireless interference into the optimization formulation, a conflict graph $\mathcal{F}$ can be defined [3]-[5], whose vertices correspond to the links in the network graph $\mathcal{G}$. There is an edge between the vertices $l_{i j}$ and $l_{p q}$ in $\mathcal{F}$ if the two links interfere with each other. The interference relationships among the links are defined by the interference model. When the CSMA/CA MAC protocol (e.g., the $802.11 \mathrm{DCF}$ ) is applied, a successful transmission requires that both the sender and the receiver are free of interference from other nodes. This condition reflects the fact that 802.11 may perform virtual carrier sensing using a request-to-send/clear-to-send (RTS/CTS) exchange and the sender must successfully receive the linklayer acknowledgement returned by the receiver. According to such an interference model, we draw an edge in the conflict graph between vertices $l_{i j}$ and $l_{p q}$ if $d_{a b} \leq \chi_{a}^{\prime}$ for $a b=$ $i q, q i, i p, p i, j p, p j, j q$ or $q j$.

Given a conflict graph $\mathcal{F}$, let $\mathcal{I}_{1}, \mathcal{I}_{2}, \ldots, \mathcal{I}_{K}$ denote all the $K$ MISs in $\mathcal{F}$ and $\alpha_{u}\left(0 \leq \alpha_{u} \leq 1\right)$ denote the fraction of time allocated to the independent set $\mathcal{I}_{u}, u=1, \ldots, K$. We can then add the constraints given in (12) and (13) to the basic MCF formulation to obtain the maximum throughput of a wireless backhaul network as

$$
\begin{aligned}
& \sum_{u=1}^{K} \alpha_{u} \leq 1 \\
& \sum_{(\nu, \eta)} x_{\nu \eta}(i, j) \leq \sum_{u: l_{i j} \in \mathcal{I}_{u}} \alpha_{u} C_{i j} \quad \forall l_{i j} \in \mathcal{L} .
\end{aligned}
$$

The MIS-based solution gives a lower bound to the network capacity [3]. The capacity is achievable by the scheduling that each MIS takes turns to transmit, with the proportion of transmission time of each set specified by $\alpha_{u}$.

An upper bound of the maximum network throughput can be solved by finding all the maximal cliques and augmenting the basic MCF formulation with the constraints that the total usage within each maximal clique is at most 1 [3]. Suppose that there are $M$ maximal cliques in the conflict graph $\mathcal{F}$, denoted as $\mathcal{C}_{1}, \ldots, \mathcal{C}_{M}$, respectively. If a link $l_{i j}$ within a maximal clique is allocated the transmission time of $t_{i j}$ during the whole clique transmission interval $T$, we can express the effective flow rate over link $l_{i j}$ as $\sum_{(\nu, \eta)} x_{\nu \eta}(i, j)=C_{i j} t_{i j} / T$. Because $\sum_{l_{i j} \in \mathcal{C}_{u}} t_{i j} / T \leq 1$, the clique constraint can be expressed in terms of the normalized throughput as

$$
\sum_{l_{i j} \in \mathcal{C}_{u}} \frac{1}{C_{i j}} \sum_{(\nu, \eta)} x_{\nu \eta}(i, j) \leq 1 \quad u=1, \ldots, M
$$

Note that the interference constraint (13) or (14) implies the basic link constraint (11). Thus, the MCF capacity region of a wireless mesh network is defined by the basic flow constraints (7)-(10) and the augmented interference constraint (13) or (14).

\section{Capacity Region Over Carrier Sense Multiple Access With Collision AVoidance}

In this section, we develop new methods of deriving the upper and lower bounds of the backhaul network throughput over the CSMA/CA MAC. We focus on the 802.11 DCF protocol due to its wide application in wireless backhaul networks. Moreover, the optimal operation point analysis presented in Section III is rigorous for 802.11 DCF [10], which is leveraged in our analysis. 


\section{A. Upper Bound of the Capacity}

Let $\hat{X}$ denote the upper bound of the MCF throughput based on the clique constraint (14). We have the following theorem with regard to a tighter upper bound of the network throughput over the CSMA/CA MAC.

Theorem 1: An upper bound of the optimal throughput of a wireless mesh network based on a CSMA/CA MAC protocol is $\hat{F}=R_{g}^{*} \hat{X}$.

Proof: The MCF formulation based on the clique constraint of (14) gives an upper bound of the network throughput, because the clique constraint defines the necessary conditions for successful transmissions within a clique [3]. The constraint (14) independently bounds the maximum throughput over each maximal clique, implying that different cliques do not interfere with each other. Within each maximal clique, we can concentrate each link into a virtual node that contends for the channel access, because simultaneous transmissions will not be successful under the CSMA/CA protocol. With regard to such virtual nodes, the VS S-G analysis in Section III determines that the maximum normalized throughput over a maximal clique is upper bounded by $R_{g}^{*}$ when the distributed CSMA/CA MAC is applied.

We now apply the clique constraints with the maximum utilization of $R_{g}^{*}$ to the basic MCF formulation for $\max \sum_{(\nu, \eta)} \sum_{l_{\nu i} \in \mathcal{L}} f_{\nu \eta}(\nu, i)$. The flow is denoted by $f_{\nu \eta}(i, j)$ to emphasize that the formulation is to find the maximum throughput over the CSMA/CA, denoted by $\hat{F}$. The basic constraints (7)-(10) still apply here. Compared to the centralized scheduling case, the clique constraint under the CSMA/CA MAC is now expressed as

$$
\sum_{l_{i j} \in \mathcal{C}_{u}} \frac{1}{C_{i j}} \sum_{(\nu, \eta)} f_{\nu \eta}(i, j) \leq R_{g}^{*} \quad u=1, \ldots, M .
$$

If we define $x_{\nu \eta}(i, j)=f_{\nu \eta}(i, j) / R_{g}^{*}$, the new MCF formulation with (15) is then transformed back to the original cliquebased MCF formulation, assuming a centralized scheduling associated with (14). Therefore, the optimal solutions $x_{\nu \eta}^{*}(i, j)=f_{\nu \eta}^{*}(i, j) / R_{g}^{*}$ and $\hat{F}=R_{g}^{*} \hat{X}$.

Obtaining a tight lower bound through clique-based MCF formulation is much more complex than computing the upper bound. To determine an achievable lower bound, the essential issue is to find sufficient conditions that ensure the feasibility of the MCF flow allocation, for which the interference among different cliques in the multihop context has to be taken into account. We next study a new concept of CSMA/CA clique to facilitate the lower bound analysis. For simplification, in the remainder of this paper, we first analyze with the assumption that all the nodes have the same communication range $\chi$ and interference range $\chi^{\prime}(\geq \chi)$ and the carrier-sensing range equals the interference range. We then discuss how we can extend our results to the general case that nodes may have different interference ranges.

\section{B. CSMA/CA Clique and CSMA/CA Area}

A circle with a diameter of $\chi^{\prime}$ is called a CSMA/CA area. Within a CSMA/CA area, all the nodes are within the carrier-

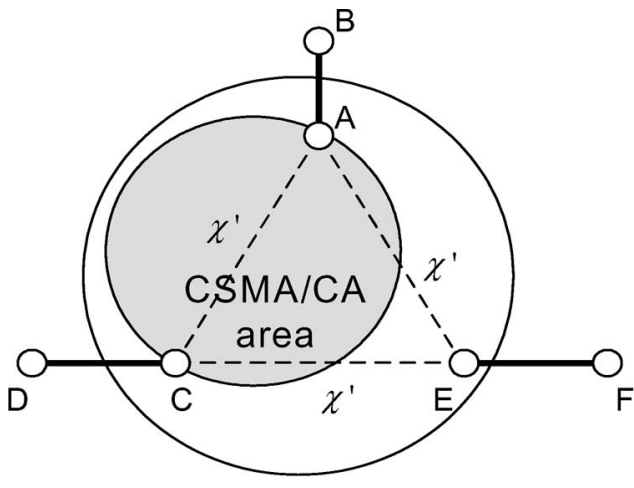

Fig. 1. CSMA/CA area, CSMA/CA clique, and maximal clique.

sensing range of each other, and the transmissions from different nodes are coordinated by the CSMA/CA protocol. It is obvious that a CSMA/CA area defines a clique, because any two links incident to nodes within the area conflict with each other. The clique is called the clique associated with the CSMA/CA area or a CSMA/CA clique for convenience. Note that a CSMA/CA area may not be large enough to cover a maximal clique. One example is illustrated in Fig. 1, where links $l_{A B}, l_{C D}$, and $l_{E F}$ form a maximal clique due to the interference between nodes $n_{A}$ and $n_{C}, n_{A}$ and $n_{E}$, and $n_{C}$ and $n_{E}$. A CSMA/CA area in this scenario can at most cover two links, and the associated CSMA/CA clique is a subset of the maximal clique.

1) Maximum Normalized Throughput: We have the following lemma with regard to the maximum normalized throughput over a CSMA/CA area and the associated CSMA/CA clique.

Lemma 1: The total normalized throughput over a CSMA/CA clique can be transformed to that over the CSMA/CA area that defines the clique, and vice versa. Thus, the maximum normalized throughput over a CSMA/CA clique is $R_{g}^{*}$.

Proof: Let $\mathcal{A}_{\mathcal{C}}$ denote the CSMA/CA area that defines a clique $\mathcal{C}$. Let $n_{i} \in \mathcal{A}_{\mathcal{C}}$ denote that node $n_{i}$ is within the CSMA/CA area $\mathcal{A}_{\mathcal{C}}$. The clique $\mathcal{C}$ consists of links $\left\{l_{i j} \mid n_{i} \in\right.$ $\mathcal{A}_{\mathcal{C}}$ or $\left.n_{j} \in \mathcal{A}_{\mathcal{C}}\right\}$. Note that it is possible that one end, in particular, the sender node of a link that belongs to the clique $\mathcal{C}$, is outside $\mathcal{A}_{\mathcal{C}}$, as shown in Fig. 2. Let $T$ denote the time interval for throughput measurement and $t_{i j}$ denote the total payload transmission time occupied by link $l_{i j}\left(l_{i j} \in \mathcal{C}\right)$ during the time interval $T$. The total normalized throughput $R_{g}$ over the clique $\mathcal{C}$ can be computed as

$$
\begin{aligned}
R_{g} & =\frac{1}{T} \sum_{l_{i j} \in \mathcal{C}} t_{i j} \\
& =\frac{1}{T}\left[\sum_{\substack{l_{i j} \in \mathcal{C} \\
n_{i} \in \mathcal{A}_{\mathcal{C}}, n_{j} \in \mathcal{A}_{\mathcal{C}}}} t_{i j}+\sum_{\substack{l_{i j} \in \mathcal{C} \\
n_{i} \in \mathcal{A}_{\mathcal{C}}, n_{j} \notin \mathcal{A}_{\mathcal{C}}}} t_{i j}+\sum_{\substack{l_{i j} \in \mathcal{C} \\
n_{i} \notin \mathcal{A}_{\mathcal{C}}, n_{j} \in \mathcal{A}_{\mathcal{C}}}} t_{i j}\right] .
\end{aligned}
$$

Considering that, in a clique, only one successful transmission is allowed at a moment, the transmission time occupied by the sender node is also equivalently occupied by the receiver 


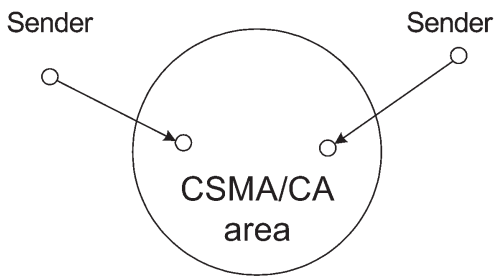

Fig. 2. Sender of a link outside of a CSMA/CA area.

node. Moreover, a link $l_{i j} \in \mathcal{C}$ always has a corresponding link $l_{j i} \in \mathcal{C}$. Then, we can continue (16) with

$$
\begin{aligned}
R_{g} & =\frac{1}{T}\left[\sum_{\substack{l_{i j} \in \mathcal{C} \\
n_{i} \in \mathcal{A}_{\mathcal{C}}, n_{j} \in \mathcal{A}_{\mathcal{C}}}} t_{i j}+\sum_{\substack{l_{i j} \in \mathcal{C} \\
n_{i} \in \mathcal{A}_{\mathcal{C}}, n_{j} \notin \mathcal{A}_{\mathcal{C}}}}\left(t_{i j}+t_{j i}\right)\right] \\
& =\frac{1}{T} \sum_{l_{i j} \in \mathcal{C}, n_{i} \in \mathcal{A}_{\mathcal{C}}} t_{i j}^{\prime}=\frac{1}{T} \sum_{i: n_{i} \in \mathcal{A}_{\mathcal{C}}} \sum_{j: l_{i j} \in \mathcal{C}} t_{i j}^{\prime} \\
& =\sum_{i: n_{i} \in \mathcal{A}_{\mathcal{C}}} \frac{t_{n_{i}}}{T}
\end{aligned}
$$

where

$$
\begin{aligned}
t_{i j}^{\prime} & = \begin{cases}t_{i j}, & \text { if } l_{i j} \in \mathcal{C}, n_{i} \in \mathcal{A}_{\mathcal{C}}, n_{j} \in \mathcal{A}_{\mathcal{C}} \\
t_{i j}+t_{j i}, & \text { if } l_{i j} \in \mathcal{C}, n_{i} \in \mathcal{A}_{\mathcal{C}}, n_{j} \notin \mathcal{A}_{\mathcal{C}}\end{cases} \\
t_{n_{i}} & =\sum_{j: l_{i j} \in \mathcal{C}} t_{i j}^{\prime}
\end{aligned}
$$

and $t_{n_{i}}$ represents the transmission time occupied by each node $n_{i} \in \mathcal{A}_{\mathcal{C}}$. The results in (18) and (19) show that the transmission time allocation in a CSMA/CA clique $\mathcal{C},\left\{t_{i j} \mid l_{i j} \in \mathcal{C}\right\}$ can be transformed to a transmission time allocation in the associated CSMA/CA area $\mathcal{A}_{\mathcal{C}},\left\{t_{n_{i}} \mid n_{i} \in \mathcal{A}_{\mathcal{C}}\right\}$. On the other hand, given a transmission allocation in $\mathcal{A}_{\mathcal{C}}$, if the destination node for each transmission is also known, the allocation then defines a transmission time allocation for the corresponding CSMA/CA clique $\mathcal{C}$. Such an equivalent relationship, in terms of the total normalized throughput, between the CSMA/CA area $\mathcal{A}_{\mathcal{C}}$ and the associated clique $\mathcal{C}$ implies that the maximum throughput over a CSMA/CA clique equals $R_{g}^{*}$ under the CSMA/CA MAC protocol.

2) Achievability of Heterogeneous Rates: Although Lemma 1 indicates that the maximum throughput over a clique can equivalently be obtained by investigating a CSMA/CA area, the basic 802.11 DCF MAC assumes that each node is equipped with the same backoff schemes and thus grab a fair share of the capacity through channel contention [38]. However, for network dimensioning, the MCF solution normally allocates different rates to flows that traverse a clique. Let $r_{i}$ denote the normalized rate of a sending node $n_{i}$. We have the following lemma with regard to the heterogeneous rate allocation.

Lemma 2: For 802.11 DCF MAC, any rate allocation over a single-hop CSMA/CA area $\mathcal{A}_{\mathcal{C}}$ that satisfiies

$$
\sum_{i: n_{i} \in \mathcal{A}_{\mathcal{C}}} r_{i} \leq R_{g}^{*}
$$

is achievable by differentiating the channel occupation time upon a successful transmission, i.e., the TXOP, for different sender nodes.

Proof: The proof is established by constructing a method of achieving any given rate allocation that satisfies (20). We assume that the initial contention window used for backoff is configurable to achieve the maximum resource utilization, as suggested in [39]. We also assume that each node always has data available at the network layer for transmission. Define that a CSMA/CA area $\mathcal{A}$ covers $N_{\mathcal{A}}$ nodes (due to the clear context, we drop the subscript $\mathcal{C}$ in this proof for easier demonstration). In a homogenous case, all the nodes have the same contention window and, thus, the same channel access probability. According to Section III, the optimal operation point can be mapped to an initial backoff window size for each node to achieve a channel access probability of $\tau=G^{*} / N_{\mathcal{A}}$ at each idle slot.

To demonstrate the achievability of the heterogeneous rate allocation of (20), we consider the borderline case with target rate allocation as

$$
\sum_{i: n_{i} \in \mathcal{A}} r_{i}=R_{g}^{*}
$$

Without loss of generality, assume that node $n_{1}$ takes the minimum rate $r_{1}=\min _{i: n_{i} \in \mathcal{A}}\left(r_{i}\right)$ and $r_{i}=\beta_{i} r_{1}\left(\beta_{i} \geq 1 ; \beta_{i} \in\right.$ $\mathbb{Z}^{+}$, the set of positive integers. Set $\beta_{1}=1$. We have

$$
r_{i}=R_{g}^{\prime} \frac{\beta_{i}}{\sum_{i: n_{i} \in \mathcal{A}} \beta_{i}}
$$

according to the rate allocation (21).

The TXOP differentiation scheme is now applied. After grabbing the channel, node $n_{i}$ is allowed to continuously send $\beta_{i}$ packets. Note that, in $802.11 \mathrm{DCF}$, the backoff counter is frozen when the channel is busy; therefore, the TXOP differentiation will not impact the fairness in accessing the channel. Let all the nodes still use the same contention window configuration; therefore, a successful channel access may be from node $n_{i}$ with probability $1 / N_{\mathcal{A}}$. Thus, the average successful transmission time is

$$
\overline{T_{s}}=\frac{1}{N_{\mathcal{A}}} \sum_{i: n_{i} \in \mathcal{A}} \beta_{i} T_{s}
$$

Consider that the initial contention window size $W_{c}$ is tuned to achieve the optimal operating point $G^{*}$. The channel-idling probability, successful transmission probability, and collision probability still maintain the values of $p_{d}^{*}, p_{s}^{*}$, and $p_{c}^{*}$, respectively, because the TXOP differentiation is transparent to the backoff operation. Define $\overline{t_{p}}=\left(1 / N_{\mathcal{A}}\right) \sum_{i: n_{i} \in \mathcal{A}} \beta_{i}\left(L_{p} / C\right)$. The channel utilization and the normalized throughput under the TXOP differentiation case, denoted as $R_{s}^{x}$ and $R_{g}^{x}$, respectively, can then be expressed as

$$
\begin{aligned}
& R_{s}^{x}=\frac{p_{s}^{*} \overline{T_{s}}}{p_{d}^{*} \sigma+p_{s}^{*} \overline{T_{s}}+p_{c}^{*} T_{c}} \\
& R_{g}^{x}=\frac{R_{s}^{x} \overline{t_{p}}}{\overline{T_{s}}}=\frac{R_{s}^{x} \frac{1}{N_{\mathcal{A}}} \sum_{i: n_{i} \in \mathcal{A}} \beta_{i} \frac{L_{p}}{C}}{\frac{1}{N_{\mathcal{A}}} \sum_{i: n_{i} \in \mathcal{A}} \beta_{i} T_{s}}=\frac{R_{s}^{x} t_{p}}{T_{s}} .
\end{aligned}
$$




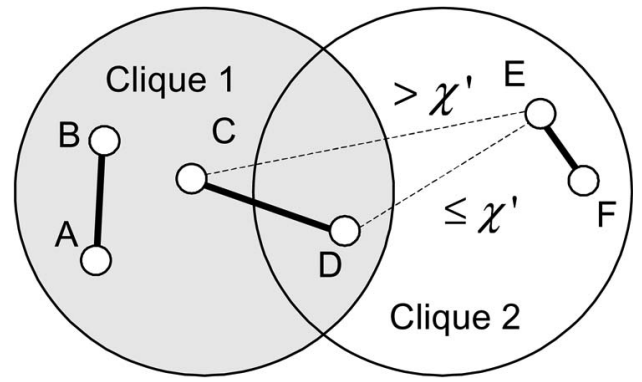

Fig. 3. Interference between two cliques.

Note that the collision duration $T_{c}$ is not impacted by the TXOP, because the collision can immediately be determined if the acknowledgement of the first transmission is not successfully returned. It is obvious that $R_{s}$ monotonically increases with $T_{s}$. Therefore, we have $R_{s}^{x} \geq R_{s}^{*}$ due to $\overline{T_{s}} \geq T_{s}$. Correspondingly, $R_{g}^{x} \geq R_{g}^{*}$. The normalized throughput of node $n_{i}$ can be computed as

$$
\begin{aligned}
R_{g, i}^{x} & =\frac{\frac{1}{N_{\mathcal{A}}} p_{s}^{*} \beta_{i} t_{p}}{p_{d}^{*} \sigma+p_{s}^{*} \overline{T_{s}}+p_{c}^{*} T_{c}}=\frac{R_{s}^{x} \beta_{i} t_{p}}{\overline{T_{s}} N_{\mathcal{A}}}=\frac{R_{s}^{x} t_{p} \beta_{i}}{T_{s} \sum_{i: n_{i} \in \mathcal{A}} \beta_{i}} \\
& =R_{g}^{x} \frac{\beta_{i}}{\sum_{i: n_{i} \in \mathcal{A}} \beta_{i}} \geq R_{g}^{*} \frac{\beta_{i}}{\sum_{i: n_{i} \in \mathcal{A}} \beta_{i}}=r_{i}
\end{aligned}
$$

That is, the TXOP differentiation can satisfy the rate requirement of (21) and, thus, of (20).

Because TXOP differentiation is transparent to the backoff operation, as exploited in the proof for Lemma 2, it is not difficult to prove another lemma.

Lemma 3: For 802.11 DCF MAC, a total throughput $R_{g}$ achievable over a single-hop CSMA/CA area under homogeneous channel contention (where each node uses the same backoff parameters and TXOP) is also achievable under any specified heterogeneous rate allocation by applying the TXOP differentiation scheme.

\section{Lower Bound of the Capacity}

The fundamental reason that the clique-based MCF formulation gives an upper bound, which may not be achievable, of the network capacity over the CSMA/CA MAC, is that the interference among the cliques in the multihop context cannot be described by the clique constraint (15). Fig. 3 illustrates the interference between two cliques, where links $l_{A B}$ and $l_{C D}$ belong to clique $\mathcal{C}_{1}$, and links $l_{C D}$ and $l_{E F}$ belong to clique $\mathcal{C}_{2}$. The interference between $l_{C D}$ and $l_{E F}$ is due to $d_{D E} \leq \chi^{\prime}$. Assume that, at a certain moment, within clique $\mathcal{C}_{1}$, only node $n_{C}$ is trying to access the channel. However, if the link $l_{E F}$ in clique $\mathcal{C}_{2}$ is transmitting at this moment, node $n_{C}$ will not be able to utilize this TXOP, although allowed in clique $\mathcal{C}_{1}$. In particular, $n_{C}$ senses an idle channel and decides to transmit (because both nodes $n_{E}$ and $n_{F}$ are out of the carrier sensing range of $n_{C}$ ), but the packet delivered to node $n_{D}$ will be corrupted due to the transmission on link $l_{E F}$. Such an interference scenario, as illustrated in Fig. 3, is called the hidden-node effect in the literature [41], [42].

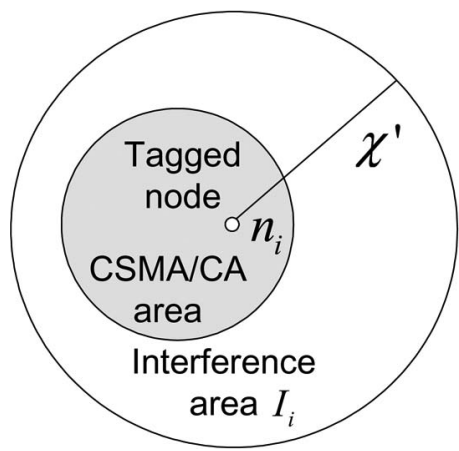

Fig. 4. Possible interference on a tagged node.

To obtain an achievable throughput, i.e., a lower bound, based on the clique model, the essence is to constrain the maximum throughput over each clique with the interclique interference taken into account. A CSMA/CA clique is defined as a maximal CSMA/CA clique (M-CSMA/CA clique) if it is not a subset of any other CSMA/CA clique. The CSMA/CA area that covers a maximal CSMA/CA clique is defined as a maximal CSMA/CA area. Let $\mathcal{C}^{s}$ denote a M-CSMA/CA clique and $R_{g}\left(\mathcal{C}^{s}\right)$ denote the total throughput over the clique. Let $N_{\mathcal{C}^{s}}$ denote the number of nodes covered by the M-CSMA/CA area $\mathcal{A}_{\mathcal{C}^{s}}$. Furthermore, let $Y\left(n_{i}\right)$ denote the maximum number of nodes that may interfere with node $n_{i}$, called the conflict degree of node $n_{i}$. We have the following lemma with regard to a sufficient condition for achievable throughput.

Lemma 4: One sufficient condition for computing the achievable MCF throughput over the network is that the throughput $R_{g}\left(\mathcal{C}^{s}\right)$ for every M-CSMA/CA clique $\mathcal{C}^{s}$ satisfies

$$
R_{g}\left(\mathcal{C}^{s}\right) \leq \frac{N_{\mathcal{C}^{s}}}{\max _{n_{i} \in \mathcal{N}}\left\{Y\left(n_{i}\right)\right\}+1} R_{g}^{*} \quad \forall \mathcal{C}^{s} .
$$

A more strict but clique-independent sufficient condition is then

$$
R_{g}\left(\mathcal{C}^{s}\right) \leq \frac{\min _{\mathcal{C}^{s}}\left\{N_{\mathcal{C}^{s}}\right\}}{\max _{n_{i} \in \mathcal{N}}\left\{Y\left(n_{i}\right)\right\}+1} R_{g}^{*} \quad \forall \mathcal{C}^{s} .
$$

Proof: Note that the throughput constraint over all the M-CSMA/CA cliques implies the constraints over the nonmaximal cliques; therefore, we only consider M-CSMA/CA cliques. Furthermore, Lemma 1 states that the total throughput over a CSMA/CA clique can be equivalent to that over the CSMA/CA area that defines the clique; thus, we prove achievable nodebased flow allocation over an M-CSMA/CA area. We use a constructive method to demonstrate that any flow allocation under (27) and (28) is feasible.

Let us consider a tagged sender node $n_{i}$. It is shown that all the nodes that may interfere with $n_{i}$ are within the circle $I_{i}$ centered at the tagged node with a radius of $\chi^{\prime}$, which is called an interference area and shown in Fig. 4. The number of nodes that interfere $n_{i}$ in its interference area is denoted as $Y\left(n_{i}\right)$. Considering the worst case, a given node might have $\max _{n_{i} \in \mathcal{N}}\left\{Y\left(n_{i}\right)\right\}$ interfering nodes within its interference area. Assuming the homogeneous case, if each node takes the same backoff configuration that gives a channel 
access probability $G^{*} /\left(\max _{n_{i} \in \mathcal{N}}\left\{Y\left(n_{i}\right)\right\}+1\right)$ and exploits the same TXOP, then any tagged node can achieve a throughput $\geq R_{g}^{*} /\left(\max _{n_{i} \in \mathcal{N}}\left\{Y\left(n_{i}\right)\right\}+1\right)$. The lower bound is achieved when all the nodes (including the tagged node) inside the worst case interference area interfere with each other (see Section III). Based on such a homogeneous construction, we can see that, for any M-CSMA/CA $\mathcal{A}_{\mathcal{C}^{s}}$ with $N_{\mathcal{C}^{s}}$ nodes, the total throughput over the area $\geq N_{\mathcal{C}^{s}} R_{g}^{*} /\left(\max _{n_{i} \in \mathcal{N}}\left\{Y\left(n_{i}\right)\right\}+1\right)$. Thus, the M-CSMA/CA throughputs specified by (27) and (28) are feasible.

We need to further consider that the MCF solution normally gives heterogeneous rate allocation to different sending nodes under the clique or area constraints (27) and (28). For any two nodes $n_{i}$ and $n_{j}$, assume that their rate allocations $r_{i}$ and $r_{j}$, transformed from the MCF solution (by Lemma 1), are related as $\left(r_{i} / r_{j}\right)=\left(\beta_{i} / \beta_{j}\right), \beta_{i}, \beta_{j} \in \mathbb{Z}^{+}$. Then, a node $n_{i}$ could be configured with a backoff window that gives a channel access probability $G^{*} /\left(\max _{n_{i} \in \mathcal{N}}\left\{Y\left(n_{i}\right)\right\}+1\right)$ and a TXOP of $\beta_{i}$ time units. Based on the analysis given in the proof of Lemma 2, we can see that such a heterogeneous TXOP configuration can ensure any MCF rate allocation under (27) and (28).

Based on Lemma 4, we can establish the following theorem with regard to the total throughput over the whole network.

Theorem 2: For a given wireless mesh network, an achievable lower bound of the maximum throughput over the CSMA/CA MAC is $\widetilde{F}=\gamma \hat{F} \geq \gamma F^{*}$, where $\hat{F}$ is the upper bound given in Theorem $1, F^{*}$ is the maximum (or optimal) capacity over CSMA/CA, and $\gamma=$ $\min _{\mathcal{C}^{s}}\left\{N_{\mathcal{C}^{s}}\right\} /\left(\max _{n_{i} \in \mathcal{N}}\left\{Y\left(n_{i}\right)\right\}+1\right)$. That is, the achievable capacity is at least $\min _{\mathcal{C}^{s}}\left\{N_{\mathcal{C}^{s}}\right\} /\left(\max _{n_{i} \in \mathcal{N}}\left\{Y\left(n_{i}\right)\right\}+1\right)$ of the maximum capacity $F^{*}$.

Proof: Let $\mathcal{C}^{s}$ denote a M-CSMA/CA clique. Suppose that there altogether exist $K$ different M-CSMA/CA cliques in the network, denoted as $\mathcal{C}_{1}^{s}, \ldots, \mathcal{C}_{K}^{s}$, respectively. To obtain an achievable throughput, we can solve the MCF problem $\max \sum_{(\nu, \eta)} \sum_{l_{\nu i} \in \mathcal{L}} f_{\nu \eta}(\nu, i)$ under the sufficient condition for flow allocation as

$$
\begin{aligned}
\sum_{l_{i j} \in \mathcal{C}_{k}^{s}} \frac{1}{C_{i j}} \sum_{(\nu, \eta)} f_{\nu \eta}(i, j) & \leq \frac{\min _{\mathcal{C}^{s}}\left\{N_{\mathcal{C}^{s}}\right\}}{\max _{n_{i} \in \mathcal{N}}\left\{Y\left(n_{i}\right)\right\}+1} R_{g}^{*} \\
& =\gamma R_{g}^{*} \quad k=1, \ldots, K
\end{aligned}
$$

in accordance with Lemma 4. Note that, by default, the flow conservation constraint, source/destination constraint, link constraint, and nonnegative constraint apply, which are not repeated in the proof for conciseness.

Let $\mathcal{Q}$ denote the set that includes all the maximal cliques, i.e., $\mathcal{Q}=\left\{\mathcal{C}_{u}, u=1, \ldots, M\right\}$, and $\mathcal{Q}^{s}$ denote the set that includes all the M-CSMA/CA cliques, i.e., $\mathcal{Q}^{s}=\left\{\mathcal{C}_{k}^{s}, k=\right.$ $1, \ldots, K\}$. It is shown that

$$
\forall \mathcal{C}_{k}^{s} \in \mathcal{Q}^{s} \Rightarrow \exists \mathcal{C}_{u(k)} \in \mathcal{Q} \text { that } \mathcal{C}_{k}^{s} \subseteq \mathcal{C}_{u(k)}
$$

according to the definition of maximal clique. Therefore

$$
l_{i j} \in \mathcal{C}_{k}^{s} \Rightarrow l_{i j} \in \mathcal{C}_{u(k)}
$$

Furthermore, we have

$$
\sum_{l_{i j} \in \mathcal{C}_{k}^{s}} \frac{1}{C_{i j}} \sum_{(\nu, \eta)} f_{\nu \eta}(i, j) \leq \sum_{l_{i j} \in \mathcal{C}_{u(k)}} \frac{1}{C_{i j}} \sum_{(\nu, \eta)} f_{\nu \eta}(i, j) .
$$

Based on (30) and (32), we can obtain that, if

$$
\sum_{l_{i j} \in \mathcal{C}_{u}} \frac{1}{C_{i j}} \sum_{(\nu, \eta)} f_{\nu \eta}(i, j) \leq \gamma R_{g}^{*}, \quad u=1, \ldots, M
$$

is satisfied, then the sufficient condition of (29), which ensures achievability, is also satisfied. That is, the maximum throughput $\widetilde{F}$ solved from the MCF formulation under (33) is also achievable. According to the similar transform used in the proof for Theorem 1, e.g., define $\gamma R_{g}^{*} x_{\nu \eta}(i, j)=f_{\nu \eta}(i, j)$, we can get $\widetilde{F}=\gamma \hat{F}$. Furthermore, let $F^{*}$ denote the maximum achievable capacity over the CSMA/CA MAC; then, the upper bound $\hat{F} \geq F^{*}$. Thus, $\widetilde{F} \geq \gamma F^{*}$. As a side benefit of our proof, the maximal-clique-based condition (33) is also a sufficient condition that ensures achievability.

Remark 2: Based on the proof of Theorem 2, we could also obtain the relationship between our capacity lower bound and the maximum capacity under the optimal centralized scheduling. Let $\hat{X}$ and $X^{*}$ denote the upper bound and the maximum capacity under the centralized scheduling, respectively. The proof of Theorem 2 states that the achievable lower bound $\widetilde{F}=\gamma R_{g}^{*} \hat{X} \geq \gamma R_{g}^{*} X^{*}$. We thus have the following corollary.

Corollary 1: For a given wireless mesh network, the lower bound of the MCF capacity computed under (33) achieves at least a fraction $\gamma R_{g}^{*}$ of the maximum capacity under the optimal centralized scheduling.

We would like to emphasize that Theorem 2 generally applies to any given network topology. Theoretically, the scaling factor $\min _{\mathcal{C}^{s}}\left\{N_{\mathcal{C}^{s}}\right\} /\left(\max _{n_{i} \in \mathcal{N}}\left\{Y\left(n_{i}\right)\right\}+1\right)$ can be computed. Given the interference range $\chi^{\prime}$, the value $\max _{n_{i} \in \mathcal{N}}\left\{Y\left(n_{i}\right)\right\}$ is easy to determine. By checking all the possible positions of a CSMA/CA area over the given network, we can also compute the value $\min _{\mathcal{C}^{s}}\left\{N_{\mathcal{C}^{s}}\right\}$. Such a method is theoretically possible but may not be practical, because the position of a CSMA/CA area can continuously change. How we can efficiently find all the CSMA/CA cliques is a new issue that is raised with this paper and will be investigated in our future work.

To give a more explicit demonstration of the achievable capacity, let us consider a uniform network in which all the nodes are uniformly or regularly distributed. We have the following corollary.

Corollary 2: In a uniform network, an achievable lower bound of the network capacity over the CSMA/CA MAC is $\widetilde{F}=\frac{1}{4} \hat{F} \geq \frac{1}{4} F^{*}$, i.e., $\gamma=\frac{1}{4}$.

Proof: We consider a uniform network that ignores the borderline effect, ${ }^{2}$ where the nodes are uniformly distributed

\footnotetext{
${ }^{2}$ In a CSMA/CA area that crosses the borderline of the network, there exists no node in the part outside the borderline. We can put some virtual node to form a uniformed node distribution. According to Lemma 3, the channel utilization occupied by the virtual node through channel contention can be transferred to those real nodes within the CSMA/CA area, with the achievability still maintained. Therefore, a borderline CSMA/CA area can be treated the same as a CSMA/CA area within the network.
} 
with a density of $\rho$. Note that each CSMA/CA area $\mathcal{A}_{\mathcal{C}}$ is covered by a circle with a diameter of $\chi^{\prime}$ and each interference area $I_{i}$ is covered by a circle with a radius of $\chi^{\prime}$. Let $B\left(\mathcal{A}_{\mathcal{C}}\right)$ and $B\left(I_{i}\right)$ denote the areas of these two circles, respectively. Thus, $N_{\mathcal{C}^{s}}=\rho B\left(\mathcal{A}_{\mathcal{C}^{s}}\right)=\rho \pi\left(\chi^{\prime} / 2\right)^{2}$ and $Y\left(n_{i}\right)+1=$ $\rho B\left(I_{i}\right)=\rho \pi \chi^{\prime 2}$. According to Theorem 2, $\widetilde{F}=\gamma \hat{F} \geq \gamma F^{*}$. In a uniform network, we have

$$
\gamma=\frac{\min _{\mathcal{C}^{s}}\left\{N_{\mathcal{C}^{s}}\right\}}{\max _{n_{i} \in \mathcal{N}}\left\{Y\left(n_{i}\right)\right\}+1}=\frac{\rho \pi\left(\frac{\chi^{\prime}}{2}\right)^{2}}{\rho \pi \chi^{\prime 2}}=\frac{1}{4} .
$$

Remark 3: We expect that the lower bound of $\frac{1}{4} \hat{F}$, although proved for the uniform network, could also well indicate the achievable capacity over an arbitrary network for two reasons. One reason is that a practical network could neither be too sparse due to the constraint of communication range nor too dense due to the consideration of collision; it implies that node distribution, in practice, will be close to uniform. The other reason is that the topology of an arbitrary network can optimally be controlled to achieve a capacity higher than that of a uniform network [17].

\section{Capacity Region in General Scenarios}

Theorems 1 and 2 give the upper and lower bounds of the network throughput, respectively, based on the MCF formulation, where the flow distribution among different ingress-egress pairs is not constrained, and multiple paths are allowed between an ingress node and an egress node. However, the linear relationship between the bounds given in the two theorems, i.e., that $\hat{F}=R_{g}^{*} \hat{X}$ and $\widetilde{F}=\gamma \hat{F}$, may not apply in some scenarios with additional application constraints. Two such scenarios are the case with the ingress-egress flow constraint and the case with the single-path routing constraint.

Ingress-Egress Flow Constraint: In practice, an access network may contract a service-level agreement with the backhaul network provider to specify the throughput requirement for given ingress-egress pairs, e.g., throughput requirement $V_{\nu \eta}$ for the ingress-egress pair or the commodity $(\nu, \eta)$. In such a scenario, we can consider the MCMF problem to provision fairness. Let $\lambda$ denote the minimum fraction of service demand that the network can guarantee for each commodity. The MCMF formulation is given as

$$
\begin{aligned}
& \max \lambda \\
& \sum_{l_{\nu i} \in \mathcal{L}} f_{\nu \eta}(\nu, i) \geq \lambda V_{\nu \eta} \quad \forall(\nu, \eta) \in \Lambda
\end{aligned}
$$

with the basic flow constraints (7)-(10) and the augmented interference constraints still applied.

Single-Path Routing: The MCF solution normally gives multiple paths to each commodity flow. Because many existing routing algorithms are confined to single-path routing, it is meaningful to derive the throughput bound under the singlepath routing. Moreover, a multipath $\mathrm{MCF}$ model for a random network topology may generate too complex routes to be implemented. According to [3], we need to add the following constraints to the MCF formulation (MMF or MCMF) to enforce the single-path routing:

$$
\begin{aligned}
& f_{\nu \eta}(i, j) \leq z_{\nu \eta}(i, j) C_{i j} \\
& \forall l_{i j} \in \mathcal{L}, \forall(\nu, \eta) \in \Lambda, \text { and } z_{\nu \eta}(i, j) \in\{0,1\} \\
& \sum_{l_{i j} \in \mathcal{L}} z_{\nu \eta}(i, j) \leq 1 \quad \forall(\nu, \eta) \in \Lambda \text { and } n_{i} \in \mathcal{N} .
\end{aligned}
$$

In the two aforementioned cases, it is not difficult to check that the linear capacity relationships described in Theorem 1 and Theorem 2 do not apply due to the extra constraints incurred. However, we would like to emphasize that, in the MCF formulation, it is the basic flow constraints (7)-(10) and the augmented interference constraints (i.e., (14) for the centralized scheduling and (15) and (33) for the CSMA/CA MAC) that determine the feasible capacity region; the additional constraints incurred by specific application requirements, in fact, could be interpreted as constraints on resource allocation within the feasible capacity region. Thus, rather than focusing on the single point of the maximum throughput, we define the capacity region as the set that consists of all possible flow allocation vectors under the basic flow constraints and the augmented interference constraints. We index the links from 1 to $|\mathcal{L}|$ and index the ingress/egress commodity flows from 1 to $|\Lambda|$. Let the vector $\boldsymbol{f}_{k}=\left(f_{k}\left(l_{1}\right), \ldots, f_{k}\left(l_{|\mathcal{L}|}\right)\right)$ denote the flow allocation over each link for the commodity $k$. The capacity region could be represented by the set $\mathcal{F}=\left\{\left(\boldsymbol{f}_{1}, \ldots, \boldsymbol{f}_{|\Lambda|}\right)\right\}$. We use $\widetilde{\mathcal{F}}$ and $\hat{\mathcal{F}}$ to denote the lower and upper bounds of the capacity region over the CSMA/CA, respectively. We use $\hat{\mathcal{X}}$ to denote the upper bound of the capacity region under the centralized scheduling. Theorem 1 and Theorem 2 can be summarized into a more generalized expression.

Theorem 3: For a wireless mesh backhaul network that operates over the CSMA/CA MAC, an upper bound of the MCF capacity region is defined by (15), giving $\hat{\mathcal{F}}=R_{g}^{*} \hat{\mathcal{X}}$, and maximizing over $\hat{\mathcal{F}}$ with the extra application constraint(s) gives an upper bound of the network throughput. A lower bound of the capacity region is defined by (33), giving $\widetilde{\mathcal{F}}=\gamma \hat{\mathcal{F}}=$ $\gamma R_{g}^{*} \hat{\mathcal{X}}$, and maximizing over $\widetilde{\mathcal{F}}$ with the extra application constraint(s) gives an achievable lower bound of the network throughput.

Heterogeneous Interference Range: In the aforementioned analysis, we assume the homogeneous interference range. However, our results can readily be extended to the general case with heterogeneous interference ranges. The lower bound scaling factor $\gamma=\min _{\mathcal{C}^{s}}\left\{N_{\mathcal{C}^{s}}\right\} /\left(\max _{n_{i} \in \mathcal{N}}\left\{Y\left(n_{i}\right)\right\}+1\right)$. It is still straightforward to determine $\max _{n_{i} \in \mathcal{N}}\left\{Y\left(n_{i}\right)\right\}$, given the interference range of each node. Furthermore, we can pick the minimum interference range $\min _{n_{i} \in \mathcal{N}} \chi^{\prime}$ to define the CSMA/CA area and then determine $\min _{\mathcal{C}^{s}}\left\{N_{\mathcal{C}^{s}}\right\}$, which gives a conservative estimation of the capacity region. 

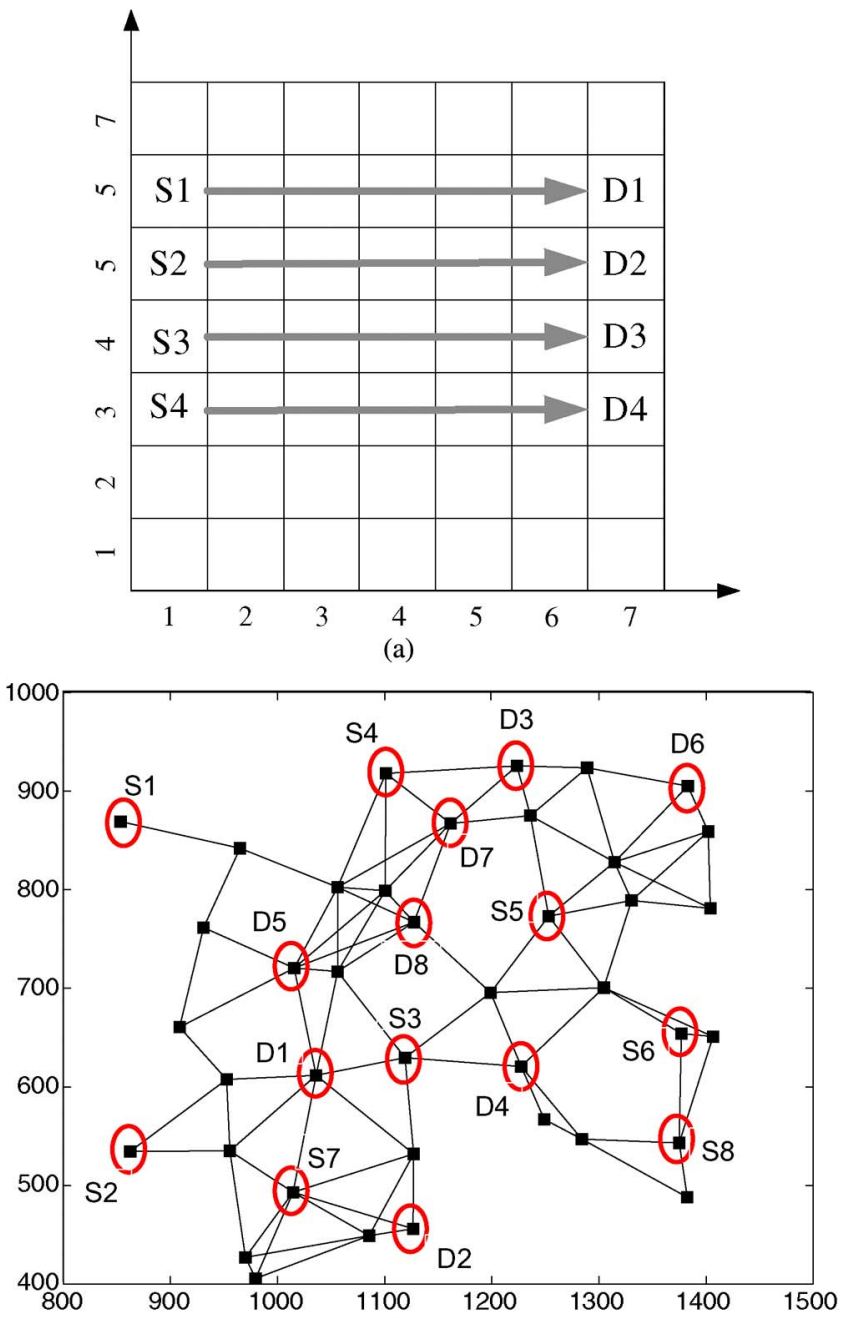

(b)

Fig. 5. Network topologies. (a) Grid topology. (b) Random topology.

\section{NumericAl Results}

In this section, we present some simulation results to validate the upper and lower bounds of the network capacity over 802.11 DCF, which are solved based on the MCF formulation. We analyze and simulate two network topologies, as shown in Fig. 5. One topology is a $7 \times 7$ grid topology over a $700 \mathrm{~m} \times 700 \mathrm{~m}$ area. The area is divided into $100 \mathrm{~m} \times 100 \mathrm{~m}$ square cells, with each cell containing one network node in its center. Four flows, as shown in Fig. 5(a), are injected into the network. The other topology is a random topology (according to a given node distribution density), as shown in Fig. 5(b), with eight pairs of ingress-egress nodes $\left(S_{i} \rightarrow D_{i}, \quad i=1, \ldots, 8\right)$ randomly selected. We adopt the algorithm used in [3] to search the MISs and maximal cliques. All the MCF problems are solved by CPLEX software [43]. We use ns-2 simulations to measure the achievable throughput over the $802.11 \mathrm{DCF}$, with routes fixed according to the MCF solution. The communication range $\chi=125 \mathrm{~m}$ and the interference range $\chi^{\prime}=275 \mathrm{~m}$.

We need to compute the optimal operation point $R_{g}^{*}$ for solving the MCF problem. The value of $R_{g}^{*}$ depends on the value of $T_{s}$ and $T_{c}$. The actual transmission time of a MAC DATA frame is $t_{D A T A}=t_{\text {header }}+L_{p} / C$, where $t_{\text {header }}$ is
TABLE I

IEEE 802.11 DCF PARAMETERS

\begin{tabular}{|l|l|}
\hline Bit rate for DATA frames & $11 \mathrm{Mbps}$ \\
\hline Bit rate for ACK frames & $1 \mathrm{Mbps}$ \\
\hline Bit rate for PLCP \& Preamble & $1 \mathrm{Mbps}$ \\
\hline Slot Time & $20 \mu \mathrm{s}$ \\
\hline SIFS & $10 \mu \mathrm{s}$ \\
\hline DIFS & $50 \mu \mathrm{s}$ \\
\hline PLCP \& Preamble & 24 bytes \\
\hline MAC header & 28 bytes \\
\hline IP header & 20 bytes \\
\hline DATA frame & $\begin{array}{l}\text { PLCP \& Preamble + MAC header } \\
+ \text { IP header + payload }\end{array}$ \\
\hline ACK fame & PLCP \& Preamble +14 bytes \\
\hline
\end{tabular}

the overhead time to transmit the headers attached in different layers. According to the 802.11 standard [37], the packet transmission time and the packet collision time are given by $T_{s}=t_{D A T A}+S I F S+A C K+D I F S$ and $T_{c}=$ $T_{s}$ for the case where RTS/CTS mechanism is not activated; on the other hand, $T_{s}=R T S+C T S+t_{D A T A}+A C K+$ $3 S I F S+D I F S$, and $T_{c}=R T S+S I F S+C T S+D I F S$ for the case where the RTS/CTS mechanism is used. Table I gives the 802.11 DCF system parameters used in our experiments.

We simulate the DCF system in both scenarios, with and without TXOP differentiation. In the case without TXOP, the payload of each packet is set as $L_{p}=1500$ bytes. In the case with TXOP, different flows will be associated with different packet size for rate differentiation. In particular, consider that the MCF solution generates $M_{\nu \eta}$ paths for the commodity $(\nu, \eta)$. Let $f_{\nu \eta}^{m}\left(m=1,2, \ldots, M_{\nu \eta}\right)$ denote the flow allocation over path $m$. We have

$$
f_{\nu \eta}=\sum_{l_{\nu i} \in \mathcal{L}} f_{\nu \eta}(\nu, i)=\sum_{m=i}^{M_{\nu \eta}} f_{\nu, \eta}^{m} \quad \forall(\nu, \eta) \in \Lambda .
$$

For TXOP differentiation, we then assign a size of $L_{\nu \eta}^{m}$ (in an integer value) to all the packets associated with the flow $f_{\nu \eta}^{m}$, and the packet sizes satisfy

$$
\begin{aligned}
\frac{L_{\nu \eta}^{m}}{L_{\nu^{\prime} \eta^{\prime}}^{m^{\prime}}} & =\frac{f_{\nu \eta}^{m}}{f_{\nu^{\prime} \eta^{\prime}}^{m^{\prime}}} \quad \forall(\nu, \eta) \in \Lambda ;\left(\nu^{\prime}, \eta^{\prime}\right) \in \Lambda ; \\
m & =1, \ldots, M_{\nu \eta} ; m^{\prime}=1, \ldots, M_{\nu^{\prime} \eta^{\prime}} . \\
\min _{(\nu, \eta), m}\left\{L_{\nu \eta}^{m}\right\} & =L_{p} .
\end{aligned}
$$

In the network, each node contends for the channel according to the DCF protocol, and each channel access may occupy a different transmission time determined by the size of the packet being served. To guarantee feasibility, the TXOP size allocation is according to the lower bound MCF solutions. Note that the flow-based TXOP differentiation according to (40) and (41) has the advantages of reducing the implementation overhead at each node and ensuring the consistency of service differentiation over the whole network. 


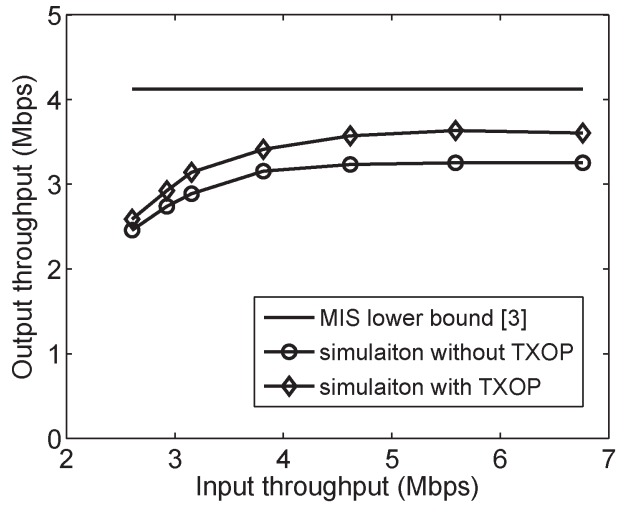

(a)

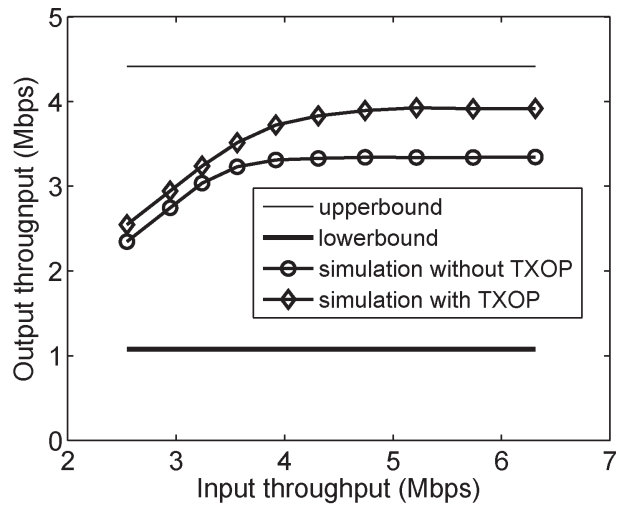

(b)

Fig. 6. Throughput in grid topology, multipath routing, without RTS/CTS, without the ingress-egress flow constraint. (a) Unachievable MIS lower bound. (b) Upper bound and achievable lower bound.

TABLE II

Service Ratio in Random Topology, With Flow Constraints, Single-Path Routing, Without RTS/CTS

\begin{tabular}{|c|l|l|l|l|}
\hline Service ratio & $\begin{array}{l}\text { With upper-bound } \\
\text { constraints }\end{array}$ & $\begin{array}{l}\text { With lower-bound } \\
\text { constraints }\end{array}$ & $\begin{array}{l}\text { Simulation without } \\
\text { TXOP }\end{array}$ & $\begin{array}{l}\text { Simulation with } \\
\text { TXOP }\end{array}$ \\
\hline$\lambda_{1}$ & 0.6515 & 0.2497 & 0.4437 & 0.5114 \\
\hline$\lambda_{2}$ & 0.8174 & 0.2270 & 0.4613 & 0.5284 \\
\hline$\lambda_{3}$ & 0.5923 & 0.2270 & 0.2385 & 0.2467 \\
\hline$\lambda_{4}$ & 0.5923 & 0.2270 & 0.3294 & 0.3395 \\
\hline$\lambda_{5}$ & 0.5923 & 0.2270 & 0.2617 & 0.2634 \\
\hline$\lambda_{6}$ & 0.5923 & 0.2270 & 0.4626 & 0.5288 \\
\hline$\lambda_{7}$ & 0.5923 & 0.2270 & 0.2275 & 0.2494 \\
\hline$\lambda_{8}$ & 0.5923 & 0.2270 & 0.3123 & 0.3728 \\
\hline $\min \lambda$ & 0.5923 & 0.2270 & 0.2275 & 0.2467 \\
\hline
\end{tabular}

\section{A. Capacity Without the Flow Constraint}

In this section, we examine the case without the ingressegress flow constraint over the grid topology. We first compute the MIS-based lower bound (MIS lower bound) for the MMF problem according to the algorithm in [3], which assumes the centralized scheduling, and examine it against simulations. For fair comparison, we deduct the constant protocol overhead from the MMF solution, that is, the MMF solution is scaled down with a factor $t_{p} / T_{s}$ (giving the net throughput of payload data) to be used as the MIS lower bound. In the simulations, we gradually increase the input rate to both examine the achievability of the lower bound and measure the maximum achievable throughput over the network. When we scale up the input rate, the rate allocation among the flows follows the ratios determined by the MMF solution.

Simulation results indicate that the MIS lower bound [3] (achievable under centralized scheduling) is infeasible over the 802.11 DCF, regardless of whether the RTS/CTS mechanism is used. The MIS lower bound is compared to the simulation results in Fig. 6(a) for the case without RTS/CTS. When the input rate is large enough, the network throughput becomes saturate, but the achieved throughput is always lower than the MIS lower bound assuming centralized scheduling. In Fig. 6(a), we observe an obviously higher throughput achieved with TXOP differentiation due to its better handling of heterogeneous rate allocation.

We then solve the upper and lower bounds based on (15) and (33), respectively. The simulation results, as presented in Fig. 6(b), show that our lower bound is achievable, because the simulation results are always above the lower bound. Note that the MMF solution with the new constraints gives different paths from those solved with the MIS constraints. ${ }^{3}$ With the new paths, the simulated throughput with TXOP differentiation is higher than that presented in Fig. 6(a). Such a phenomenon indicates that our new constraints result in paths that are more suitable for the CSMA/CA MAC than paths that are generated with the MIS-based constraints, which, however, assume a time-slotted system.

\section{B. Capacity With the Flow Constraint}

In this section, we examine the case with the ingress-egress flow constraint over the random topology through a MCMF formulation. Moreover, single-path routing is enforced according to the discussion in Section V-D. The throughput under the cases with and without RTS/CTS is compared. It can be computed that $R_{g}^{*}=0.4306$ for the case with RTS/CTS and $R_{g}^{*}=0.5601$ for the case without. We set the ingress-egress service demands for the eight commodities as [1.0, 1.0, 0.4, $0.6,0.4,1.0,0.4,0.6] \mathrm{Mb} / \mathrm{s}$. We solve the MCMF problem under (15) and (33) to obtain the upper and lower bounds, respectively. The achieved flow allocation rations with regard to each commodity demand (i.e., the service ratios) and the simulation results are presented in Table II. The simulation results are obtained by serving the input traffic with the paths

\footnotetext{
${ }^{3}$ The MMF solution generates the same set of paths for both the upper and lower bound cases due to the scaling relationship (with the factor $\gamma$ ).
} 


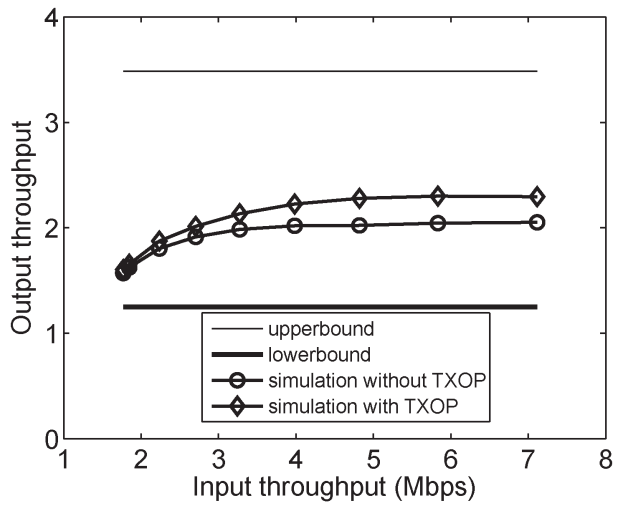

(a)

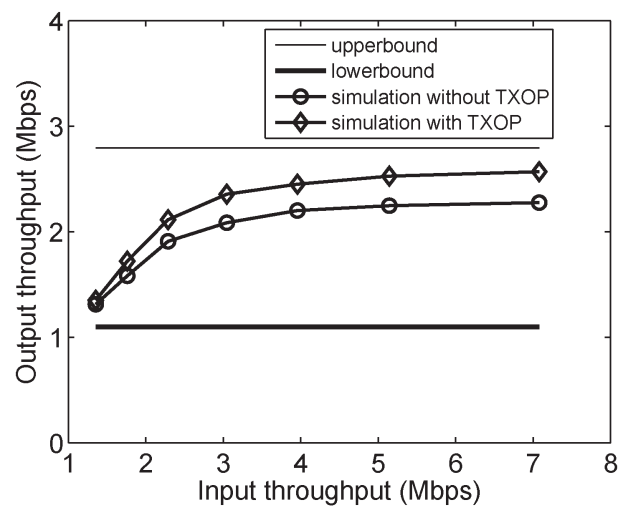

(b)

Fig. 7. Throughput in random topology, single-path routing, with the flow constraint. (a) Without RTS/CTS. (b) With RTS/CTS.

generated from the lower bound solution and taking the ratios between the achieved throughput and the corresponding service demand. The simulation results demonstrate that the achieved service ratio for each commodity is larger than the lower bound value, with a more obvious margin under TXOP differentiation.

The analytical capacity bounds and simulation results are also presented in Fig. 7 in terms of the aggregate throughput over the whole network. In both cases with and without RTS/CTS, the maximum achievable throughput is between the upper and the lower bounds, and TXOP differentiation results in better performance. We further observe that the analytical capacity bounds in the case with RTS/CTS have smaller values that in the case without RTS/CTS, whereas the simulated capacity with RTS/CTS is higher. The reason is that the analytical capacity bound is related to the optimal operation point $R_{g}^{*}$, which is achieved in the nonsaturated situation with a small collision probability. In such a low-collision situation, the RTS/CTS mechanism will perform worse than the basic access mode without RTS/CTS, because the extra bandwidth overhead due to RTS/CTS communications overweighs its benefit in mitigating the collision cost. In the simulation results, however, we explore the network capacity by saturating the network with increasing input traffic. In the saturated situation, the collision probability is considerably high, where the RTS/CTS operation shows its benefit.

To examine the robustness of our network dimensioning results to topology, we simulate the throughput over different

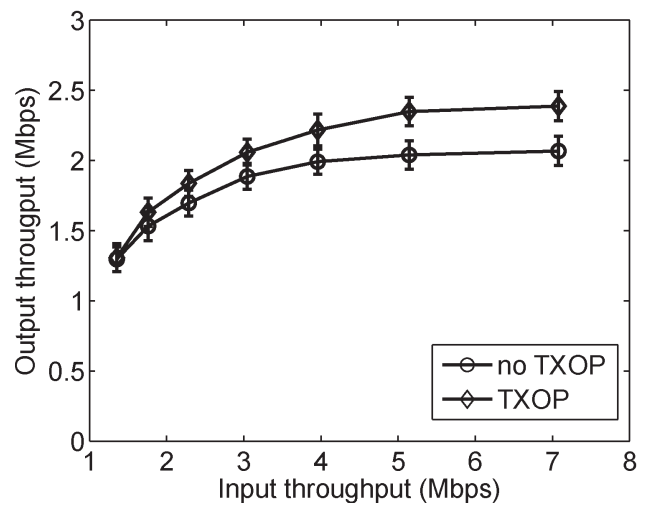

Fig. 8. Throughput over various random topologies, with RTS/CTS.

random topologies. In particular, we fix the positions of all the source destination nodes and the positions of all the nodes on the used paths, because they are the dimensioning or planning results, but we randomly change the positions of other nodes around. We simulated 50 topologies. The simulated throughput curves (averaged over all the topologies) versus the input rate and the associated $90 \%$ confidence intervals are plotted in Fig. 8, with the RTS/CTS operation turned on in both cases with and without TXOP. The small confidence intervals indicate that the impact due to the topology variation is not significant. The reason is that, in a well-connected network, the optimization under the interference constraint will well distribute the traffic over the whole area for the highest throughput. Random topology variation over the whole area will not much impact the planning results.

\section{CONCLUSION}

This paper addressed the issue that the existing capacity studies of wireless backhaul networks, based on MCF formulation, assume impractical centralized scheduling and generate capacity region that is unachievable over a practical CSMA/CA MAC protocol. The fundamental contribution of this paper is to derive necessary and sufficient conditions for MCF flow allocation, which take into account CSMA/CA randomness and therefore generate a tight upper bound and an achievable lower bound of the network capacity over the CSMA/CA MAC. Toward our objective, we have integrated the CSMA/CA MAC analysis with the MCF formulation, defined a new concept of CSMA/CA clique, and developed a method of analyzing interclique interferences, which are interesting results by themselves. In future work, we will study how the lower bound of the capacity region can further be improved.

\section{REFERENCES}

[1] W. Wang, X. Liu, and D. Krishnaswamy, "Robust routing and scheduling in wireless mesh networks," in Proc. IEEE SECON, 2007, pp. 471-480.

[2] L. Badia, A. Erta, L. Lenzini, and M. Zorzi, "A general interferenceaware framework for joint routing and link scheduling in wireless mesh networks," IEEE Netw., vol. 22, no. 1, pp. 32-38, Jan./Feb. 2008.

[3] K. Jain, J. Padhye, V. Padmanabhan, and L. Qiu, "Impact of interference on multihop wireless network performance," in Proc. ACM MobiCom, 2003, pp. 66-80.

[4] T. Nandagopal, T.-E. Kim, X. Gao, and V. Bharghavan, "Achieving MAClayer fairness in wireless packet networks," in Proc. ACM MobiCom, 2000, pp. 87-98. 
[5] K. N. Ramachandran, E. M. Belding, K. C. Almeroth, and M. M. Buddhikot, "Interference-aware channel assignment in multiradio wireless mesh networks," in Proc. IEEE INFOCOM, 2006, pp. 1-12.

[6] V. S. Anil Kumar, M. V. Marathe, and S. Parthasarathy, "Algorithmic aspects of capacity in wireless networks," in Proc. ACM SIGMETRICS, 2005, pp. 133-144.

[7] H. Viswanathan and S. Mukherjee, "Throughput-range tradeoff of wireless mesh backhaul networks," IEEE J. Sel. Areas Commun., vol. 24, no. 3, pp. 593-602, Mar. 2006.

[8] P.-J. Wan, "Multiflows in multihop wireless networks," in Proc. ACM MobiHoc, 2009, pp. 85-94.

[9] Y. Wang, W. Wang, X.-Y. Li, and W.-Z. Song, "Interference-aware joint routing and TDMA link scheduling for static wireless networks," IEEE Trans. Parallel Distrib. Syst., vol. 19, no. 2, pp. 1709-1726, Dec. 2008.

[10] Y. Cheng, X. Ling, and W. Zhuang, "A protocol-independent approach for analyzing the optimal operation point of CSMA/CA protocols," in Proc. IEEE INFOCOM, Apr. 2009, pp. 2070-2078.

[11] R. Gupta, J. Musacchio, and J. Walrand, "Sufficient rate constraints for QoS flows in ad hoc networks," Ad Hoc Netw., vol. 5, no. 4, pp. 429-443, May 2007.

[12] G. Bianchi, I. Tinnirello, and L. Scalia, "Understanding 802.11e contention-based prioritization mechanisms and their coexistence with legacy 802.11 stations," IEEE Netw., vol. 19, no. 4, pp. 28-34, Jul./Aug. 2005.

[13] M. Alicherry, R. Bhatia, and L. Li, "Joint channel assignment and routing for throughput optimization in multiradio wireless mesh networks," in Proc. ACM MobiCom, 2005, pp. 58-72.

[14] X. Wu, R. Srikant, and J. R. Perkins, "Scheduling efficiency of distributed greedy scheduling algorithms in wireless networks," IEEE Trans. Mobile Comput., vol. 6, no. 6, pp. 595-605, Jun. 2007.

[15] P. Chaporkar, K. Kar, X. Luo, and S. Sarkar, "Throughput and fairness guarantees through maximal scheduling in wireless networks," IEEE Trans. Inf. Theory, vol. 54, no. 2, pp. 572-594, Feb. 2008.

[16] C. Joo, X. Lin, and N. B. Shroff, "Understanding the capacity region of the greedy maximal scheduling algorithm in multihop wireless networks," IEEE/ACM Trans. Netw., vol. 17, no. 4, pp. 1132-1145, Aug. 2009.

[17] P. Gupta and P. R. Kumar, "The capacity of wireless networks," IEEE Trans. Inf. Theory, vol. 46, no. 2, pp. 388-404, Mar. 2000.

[18] A. E. Gamal, J. Mammen, B. Prabhakar, and D. Shah, "Throughput-delay tradeoff in wireless networks," in Proc. IEEE INFOCOM, Mar. 7-11, 2004, pp. 464-475.

[19] D. Shila, Y. Cheng, and T. Anjali, "Throughput and delay analysis of hybrid wireless networks with multihop uplinks," in Proc. IEEE INFOCOM, Apr. 10-15, 2011, pp. 1476-1484.

[20] Y. Wang, X. Chu, X. Wang, and Y. Cheng, "Optimal multicast capacity and delay tradeoffs in MANETs: A global perspective," in Proc. IEEE INFOCOM, Apr. 10-15, 2011, pp. 640-648.

[21] Y. Shi, Y. T. Hou, and S. Kompella, "How to correctly use the protocol interference model for multihop wireless networks," in Proc. ACM MobiHoc, May 2009, pp. 239-248.

[22] Y. Cheng, H. Li, P. Wan, and X. Wang, "Capacity region of a wireless mesh backhaul network over the CSMA/CA MAC," in Proc. IEEE INFOCOM (Mini Conference), Mar. 2010, pp. 1-5.

[23] K. Medepalli and F. A. Tobagi, "Towards performance modeling of IEEE802.11-based wireless networks: A unified framework and its application," in Proc. IEEE INFOCOM, 2006, pp. 1-12.

[24] M. Garetto, T. Salonidis, and E. W. Knightly, "Modeling per-flow throughput and capturing starvation in CSMA multihop wireless networks," IEEE/ACM Trans. Netw., vol. 16, no. 4, pp. 864-877, Aug. 2008.

[25] L. Georgiadis, M. J. Neely, and L. Tassiulas, "Resource allocation and cross-layer control in wireless networks," Found. Trends Netw., vol. 1, no. 1, pp. 1-144, Apr. 2006.

[26] L. Bui, A. Eryilmaz, R. Srikant, and X. Wu, "Joint asynchronous congestion control and distributed scheduling for wireless networks," in Proc. IEEE INFOCOM, 2006, pp. 1-12.

[27] X. Lin and S. Rasool, "A distributed joint channel-assignment, scheduling, and routing algorithms for multichannel ad hoc wireless networks," in Proc. IEEE INFOCOM, 2007, pp. 1118-1126.

[28] J. Liu, Y. Yi, A. Proutiere, M. Chiang, and V. Poor, "Maximizing utility via random access without message passing," Mountain View, CA, Tech. Rep. 2008-128, Sep. 2008.

[29] L. Jiang and J. Walrand, "A distributed CSMA algorithm for throughput and utility maximization in wireless networks," in Proc. Allerton Conf., Sep. 2008, pp. 1511-1519.

[30] F. Daneshgaran, M. Laddomada, F. Mesiti, and M. Mondin, "Unsaturated throughput analysis of IEEE 802.11 in presence of nonideal transmission channel and capture effects," IEEE Trans. Wireless Commun., vol. 7, no. 4, pp. 1276-1286, Apr. 2008.

[31] G. J. Sutton, R. Liu, X. Yang, and I. B. Collings, "Modeling capture effect for 802.11 DCF under Raleigh fading," in Proc. IEEE ICC, May 2010, pp. 1-6.

[32] H. Wu, F. Yang, K. Tan, J. Chen, Q. Zhang, and Z. Zhang, "Distributed channel assignment and routing in multiradio multichannel multihop wireless networks," IEEE J. Sel. Areas Commun., vol. 24, no. 11, pp. 1972-1983, Nov. 2006

[33] H. Li, Y. Cheng, C. Zhou, and P. Wan, "Multidimensional-conflict-graphbased computing for optimal capacity in MR-MC wireless networks," in Proc. IEEE ICDCS, Jun. 21-25, 2010, pp. 774-783.

[34] Y. Ding, Y. Huang, G. Zeng, L. Xiao, "Using partially overlapping channels to improve throughput in wireless mesh networks," IEEE Trans. Mobile Comput. [Online]. Available: http://ieeexplore.ieee.org/ stamp/stamp.jsp?tp $=$ \&arnumber $=6042867$

[35] A. Mishra, V. Shrivastava, S. Banerjee, and W. Arbaugh, "Partially overlapped channels not considered harmful," in Proc. SIGMETRICS, 2006, pp. 63-74.

[36] H. Li, A. Srivastava, and Y. Cheng, "Computing the optimal capacity of multiradio multichannel wireless network over partially overlapping channels," in Proc. IEEE GLOBECOM, 2011, pp. 1-5.

[37] H. Zhai, X. Chen, and Y. Fang, "How well can the IEEE 802.11 wireless LAN support quality of service?" IEEE Trans. Wireless Commun., vol. 4, no. 6, pp. 3084-3094, Nov. 2005.

[38] G. Bianchi, "Performance analysis of the IEEE 802.11 distributed coordination function," IEEE J. Sel. Areas Commun., vol. 18, no. 3, pp. 535-547, Mar. 2000.

[39] Y. Cheng, X. Ling, W. Song, L. X. Cai, W. Zhuang, and X. Shen, "A crosslayer approach for WLAN voice capacity planning," IEEE J. Sel. Areas Commun., vol. 25, no. 4, pp. 678-688, May 2007.

[40] D. Bertsekas and R. Gallenger, Data Networks, 2nd ed. Englewood Cliffs, NJ: Prentice-Hall, 1992.

[41] J. Li, C. Blake, D. S. J. De Couto, H. I. Lee, and R. Morris, "Capacity of ad hoc wireless networks," in Proc. ACM MobiCom, 2001, pp. 61-69.

[42] P. C. Ng and S. C. Liew, "Throughput analysis of IEEE 802.11 multihop ad hoc networks," IEEE/ACM Trans. Netw., vol. 15, no. 2, pp. 309-322, Apr. 2007.

[43] Ilog cplex Suite. [Online]. Available: http://www.ilog.com/products/ cplex/

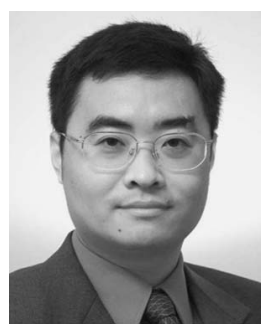

Yu Cheng (S'01-M'04-SM'09) received the B.E. and M.E. degrees in electrical engineering from Tsinghua University, Beijing, China, in 1995 and 1998, respectively, and the Ph.D. degree in electrical and computer engineering from the University of Waterloo, Waterloo, ON, Canada, in 2003.

From September 2004 to July 2006, he was a Postdoctoral Research Fellow with the Department of Electrical and Computer Engineering, University of Toronto, Toronto, ON. Since August 2006, he has been an Assistant Proffesor with the Department of Electrical and Computer Engineering, Illinois Institute of Technology, Chicago. His research interests include next-generation Internet architectures and management, wireless network performance analysis, network security, and wireless/wireline interworking.

Dr. Cheng is the recipient of a Postdoctoral Fellowship Award from the Natural Sciences and Engineering Research Council of Canada (NSERC) in 2004, the Best Paper Award at the Fourth International Conference on Heterogeneous Networking for Quality, Reliability, Security and Robustness (QShine) in 2007, the Best Paper Award at the IEEE International Conference on Communications (ICC) in 2011, and the National Science Foundation (NSF) Faculty Early Career Development (CAREER) Award in 2011. He served as Co-Chair of the Wireless Networking Symposium of IEEE ICC in 2009, the Communications QoS, Reliability, and Modeling Symposium of the IEEE Global Communications Conference (GLOBECOM) in 2011, and the Signal Processing for Communications Symposium of IEEE ICC in 2012. He was a Technical Program Committee (TPC) Co-Chair of the Sixth International Conference on Wireless Algorithms, Systems, and Applications (WASA) in 2011. He is an Associate Editor for IEEE TRANSACTIONS ON VEHICULAR Technology and a New Books and Multimedia Column Editor for IEEE NETWORK. 


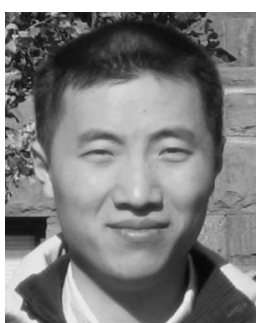

Hongkun Li (S'09) received the B.Eng. degree in information engineering from Zhejiang University, Hangzhou, China, in 2004 and the M.Eng. degree in electrical engineering from Beijing University of Post and Telecommunication, Beijing, China, in 2007. He is currently working toward the Ph.D. degree with the Department of Electrical and Computer Engineering, Illinois Institute of Technology, Chicago.

His research interests include capacity analysis and resource allocation for multiradio multichannel wireless networks and routing protocol design and analysis for wireless networks.

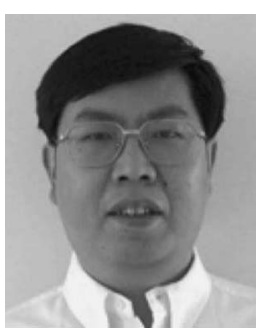

Peng-Jun Wan (M'09) received the B.S. degree from Tsinghua University, Beijing, China, the M.S. degree from the Chinese Academy of Sciences, Beijing, and the Ph.D. degree from the University of Minnesota, Minneapolis.

$\mathrm{He}$ is currently a Professor of computer science with the Department of Computer Science, Illinois Institute of Technology, Chicago. His research interests include wireless networks and algorithm design and analysis.

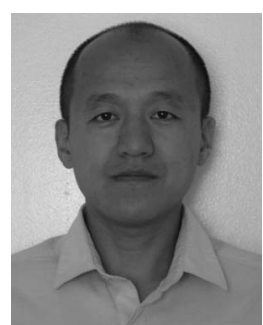

Xinbing Wang (SM'12) received the B.S. degree (with honors) in automation from Shanghai Jiao Tong University, Shanghai, China, in 1998, the M.S. degree in computer science and technology from Tsinghua University, Beijing, China, in 2001, and the $\mathrm{Ph} . \mathrm{D}$. degree, with a major in electrical and computer engineering and a minor in mathematics, from North Carolina State University, Raleigh, in 2006.

$\mathrm{He}$ is currently a Professor with the Department of Electronic Engineering, Shanghai Jiao Tong University. His research interests include resource allocation and management in mobile and wireless networks, Transmission Control Protocol asymptotics analysis, wireless capacity, cross-layer call admission control, asymptotics analysis of hybrid systems, and congestion control over wireless ad hoc and sensor networks.

Dr. Wang has been a member of the Technical Program Committees of several conferences, including the 13th ACM International Symposium on Mobile Ad Hoc Networking and Computing Conference on Mobile Computing and Networking (MobiCom) in 2012, the 13th ACM International Symposium on Mobile Ad Hoc Networking and Computing (ACM MobiHoc) in 2012, and the IEEE International Conference on Computer Communications (INFOCOM) from 2009 to 2013 . 